\title{
AN UPDATED CHECKLIST OF CROATIAN TACHINIDAE (DIPTERA)
}

\section{Erikas Lutovinovas ${ }^{1}$, Roman Ozimec ${ }^{2}$, Miroslav Barták ${ }^{3}$ \& BožE KOKAN ${ }^{4}$}

\begin{abstract}
${ }^{1}$ Nature Research Centre, Akademijos 2, LT-084 12 Vilnius, Lithuania (e-mails: wohlfahrtia@gmail.com, erikas.lutovinovas@gamtostyrimai.lt)

${ }^{2}$ ADIPA-Croatian Society for Natural History Diversity Research \& Conservation, Orehovečki ogranak 37, HR-100 40 Zagreb, Croatia (e-mails: ozimec.roman@gmail.com, roman.ozimec@adipa.com)

${ }^{3}$ Department of Zoology and Fisheries, Faculty of Agrobiology, Food and Natural Resources, Czech University of Life Sciences, Kamýcká 129, CZ-165 21 Praha, Czech Republic (e-mail: bartak@af.czu.cz)

${ }^{4}$ Natural History Museum and Zoo, Kolombatovićevo šetalište 2, HR-210 00 Split, Croatia (e-mail: boze@prirodoslovni.hr)
\end{abstract}

Lutovinovas, E., Ozimec, R., Barták, M. \& Kokan, B.: An updated checklist of Croatian Tachinidae (Diptera). Vol. 27, No. 1., 57-96, 2018, Zagreb.

We have listed 307 species of Tachinidae presently known from the country. Our checklist contains data from all available sources cited below the species names. New material consisting of 100 species has been studied to support the literature. There are still 29 doubtful records, as well as 3 doubtful taxa, mentioned by old references, the validity of which needs to be confirmed; these records are reviewed apart from the main species list.

Keywords: Tachinidae, checklist, distribution, Croatia, Balkan Peninsula

Lutovinovas, E., Ozimec, R., Barták, M. \& Kokan, B.: Ažurirani popis hrvatskih muha gusjeničarki (Diptera: Tachinidae). Vol. 27, No. 1., 57-96, 2018, Zagreb.

Utvrdili smo 307 vrsta muha gusjeničarki (Tachinidae) trenutno poznatih za Hrvatsku. Naš ažurirani popis sadrži podatke iz svih raspoloživih izvora koje citiramo nakon naziva vrste. Kao dopunu literaturnim navodima analiziran je novosakupljeni materijal koji sadrži 100 vrsta. Prisutno je 29 dvojbenih nalaza, kao i 3 taksonomski dvojbene svojte koje se navode u starim referencama, a čiju valjanost još treba potvrditi; ti nalazi se obrađuju odvojeno od glavnog popisa vrsta.

Ključne riječi: Tachinidae, popis, rasprostranjenost, Hrvatska, Balkanski poluotok

\section{INTRODUCTION}

Tachinid flies are one of the largest families in the order Diptera and distinctly the largest group of the Calyptrata section (PAPE et al., 2011). Some of these insects are black, tiny and rarely seen in nature, while others are bright, large, and easily spotted in the field. Tachinids consume their basic food requirements in the larval stage, but the adults, when visiting flowers, play a role as pollinators of some plants (ToOKen et al., 2006; Al-Dobai et al., 2012; Krivosheina \& Richter, 2015). All known species of tachinids develop as obligate endoparasitoids in many insect host orders (predominantly moth caterpillars, but also sawfly larvae, beetle larvae and adults, crane fly larvae, true bugs, crickets, etc.), and even, rarely, in several terrestrial noninsect arthropods (specifically centipedes and scorpions), and as such have great ecological importance (STIREMAN et 
al., 2006). The tachinids as a family have associations with a wide range of hosts, but certain groups of tachinids have more specialized associations. Data on host preferences for the Palaearctic species can be found in Herting (1960), Tschorsnig \& Herting (1994) or Tschorsnig (2017). However, it is not just the diversity of hosts in the habitat, but also habitat connectivity that enables parasitoid spillover, and other ecological factors determine diversity of tachinids (INCLÁn et al., 2014, 2015, 2016). Most tachinids are thermophilic, and the fauna of Southern Europe is therefore richer than that of Central Europe, where tachinids have been thoroughly studied (Tschorsnig \& HerTING, 1994). There are also some species that have expanded their distribution in Europe northwards, due to global warming or other reasons (BERgström \& BARTCH, 2005; Ziegler, 2011; FranCATi et al., 2017) or that have been introduced to Europe from other continents and become established in different countries (SALERno et al., 2002; GRoot et al., 2007; PÉtremAND et al., 2015). However, the fauna of several southeastern European countries remains relatively unknown and requires further investigation.

The most up-to-date checklist of Tachinidae of the Balkan countries (HubENov, 2008a) lists 241 species for the fauna of Croatia, which is certainly an incomplete representation of the actual fauna, when the species diversity of nearby countries is taken into account. Croatia is a country that boasts a spectacular landscape and many rare habitats, and due to its specific geographical position that covers four biogeographical regions (Pannonian, Continental, Alpine and Mediterranean), it is possibly one of the richest European countries in terms of biodiversity (RADović et al., 2009). The aim of the present publication is to provide a species list based on more complete reference sources, and to confirm some older and unreliable records, adding recent faunistic data.

\section{MATERIAL AND METHODS}

The complete list of currently known Croatian tachinid fauna is based on previously published sources as well as on our own original data. In the case of species that were not collected during recent field research, information about them is drawn from published sources; if their presence is confirmed by both new material and published sources, both sets of data are listed. We revised numerous additional sources that were ignored by Hubenov (2008a): Siebold (1843); Frauenfeld (1856; 1860); Schiner (1862); Brischke (1885); Wachtl (1889); Kuhlmann (1890); Равst (1890a; 1890b); Strobl (1893; 1898; 1904); Brauer \& Bergenstamm (1894); Langhoffer (1896; 1920); NovaK (1899); Thalhammer (1899); Riedel (1908); Tölg \& Fahringer (1911); Kovačević (1926; 1928); Baranov (1928; 1929c; 1930c; 1932); Baranov \& Hergula (1928); Burgess \& Crossman (1929); Hergula (1928; 1929); PArker et al. (1929); Dowden (1933); Melis (1940); Androić (1956; 1957); Bjegović (1957); Coe (1960; 1962); Hadžistević (1961); Keglević (1970); Herting (1974; 1975; 1985; 1993); Maceljski \& Balarin (1975); Tschorsnig (1990, 2005); Rucner (1994); Tschorsnig \& Herting (1994; 2005); Cooper \& O’Hara (1996); Ford et al. (2000); Rubin (2000); Rieger \& Tschorsnig (2001); Britvec (2000; 2004); Sun \& Marshall (2003); Cerretti (2005); MücKSTEIN et al. (2007); we also reviewed the most recent reference sources: CERretTi \& SHIMA (2011); Bystrowski (2012); Robertson \& Shaw (2012); Pernek et al. (2013; 2016); O’HAra \& Cerretti (2016); Pohjoismäki et al. (2016); Ziegler et al. (2016); Herting (2017); Tschorsnig (2017); Lutovinovas et al. (2018). These records were revised by the first and second authors of this report (E. Lutovinovas and R. Ozimec).

Most of the studied material originates from Gornji Muć (Central Dalmatia) and was collected in an abandoned garden in seasons of 2013 and 2014 using a modified Townes type Malaise trap (with a broader and higher "roof"). The trap was operated by the fo- 
urth author of this report (B. Kokan), and material selection was carried out by the third author (M. Barták). More detailed description of the habitat in Gornji Muć is provided in one of the previous studies (see BARTÁK \& KOKAN, 2017). Single specimens originating from other localities were collected by M. Kafka (2002), M. Barták (2007, 2011), M. Kadlecová (2008), and J. Halada (2009, 2013); these specimens were sampled in different parts of Dalmatia using a conventional entomological net. Voucher specimens are displayed in a collection at the Czech University of Life Sciences Prague (CULSP). Full names of the localities are presented next to each recorded species; their coordinates and collectors are provided separately (Tab. 1).

Tab. 1. Details of sampling locations with collectors of Tachinidae in Croatia

\begin{tabular}{|c|c|c|c|c|c|}
\hline No. & Locality & Altitude & County & Coordinates & Collector \\
\hline 1 & Gornji Muć & $500 \mathrm{~m}$ & Split & $43^{\circ} 41^{\prime} 27^{\prime \prime} \mathrm{N} 16^{\circ} 29^{\prime} 44^{\prime \prime} \mathrm{E}$ & B. Kokan \\
\hline 2 & Molunat & $150 \mathrm{~m}$ & Dubrovnik & $42^{\circ} 28^{\prime} 52^{\prime \prime} \mathrm{N} 18^{\circ} 25^{\prime} 57^{\prime \prime} \mathrm{E}$ & M. Barták \\
\hline 3 & Omiš & $200 \mathrm{~m}$ & Split & $43^{\circ} 26^{\prime} 00^{\prime \prime} \mathrm{N} 16^{\circ} 41^{\prime} 00^{\prime \prime} \mathrm{E}$ & J. Halada \\
\hline 4 & Paklenica & - & Zadar & $44^{\circ} 22^{\prime} 00^{\prime \prime} \mathrm{N} 15^{\circ} 26^{\prime} 00^{\prime \prime} \mathrm{E}$ & M. Kafka \\
\hline 5 & Pula & - & Istria & $44^{\circ} 52^{\prime} 00^{\prime \prime} \mathrm{N} 13^{\circ} 51^{\prime} 00^{\prime \prime} \mathrm{E}$ & J. Halada \\
\hline 6 & Senj & seashore & Lika & $44^{\circ} 58^{\prime} 26^{\prime \prime} \mathrm{N} 14^{\circ} 55^{\prime} 13^{\prime \prime} \mathrm{E}$ & M. Barták \\
\hline 7 & Vir & - & Zadar & $44^{\circ} 19^{\prime} 09^{\prime \prime} \mathrm{N} 15^{\circ} 02^{\prime} 59^{\prime \prime} \mathrm{E}$ & M. Barták \\
\hline 8 & Vodice & - & Šibenik & $43^{\circ} 45^{\prime} 00^{\prime \prime} \mathrm{N} 15^{\circ} 46^{\prime} 00^{\prime \prime} \mathrm{E}$ & M. Kadlecová \\
\hline
\end{tabular}

New material is studied to support the literature, especially older and unreliable references. The material was identified by the first author of this report (E. Lutovinovas). Species were identified in accordancewith CERRETTI (2010); however, we also consulted Mesnil (1944-1975), Herting (1983) as well as certain recent descriptions (Cerretti \& Tschorsnig, 2008; Gilasian et al., 2013; Ziegler et al., 2016). Taxonomy and general distribution were acquired primarily from Herting \& Dely-Draskovits (1993). The species of the tribe Eutherini (Euthera fascipennis) is transferred from the subfamily Phasiinae to Dexiinae in accordance with O'HARA \& Wood (2004), O'HARA et al. (2009) and Cerretti (2010). Subfamilies, tribes, genera and species are listed alphabetically within the species list, whereas all unreliable records are reviewed in Appendix 1.

\section{RESULTS}

TACHINIDAE Robineau-Desvoidy, 1830

DeXIINAe Macquart, 1834

Dexiini Macquart, 1834

\section{BILLAEA Robineau-Desvoidy, 1830}

Billaea adelpha (Loew, 1873)

Literature: Tschorsnig et al. (2004); Hubenov (2008a).

Billaea maritima (Schiner, 1862)

Literature: Tschorsnig et al. (2004); Hubenov (2008a). - Material: Gornji Muć, 14.-28. IX.2014, 1 \% . 
Billaea pectinata (Meigen, 1826)

Literature: LANGHoffer (1920); BARAnov (1928); Tschorsnig et al. (2004); Hubenov (2008a).

\section{DEXIA Meigen, 1826}

Dexia rustica (Fabricius, 1775)

Literature: LANGHOFFer (1920); BARANOv (1928); Coe (1962); Tschorsnig et al. (2004); Hubenov (2008a). - Material: Gornji Muć, 11.VIII.-15.IX.2013, 1 ㅇ.

\section{DINERA Robineau-Desvoidy, 1830}

Dinera carinifrons (Fallén, 1817)

Literature: LANGHOfFer (1920); BARANov (1928); Tschorsnig et al. (2004); Hubenov (2008a); Ziegler et al. (2016). - Material: Gornji Muć, 11.-27.IV.2014, 1 q .

Dinera ferina (Fallén, 1817) [= Myocerops ferina Fallén, 1817]

Literature: Strobl (1893); LANghoffer (1920); Baranov (1928); Coe (1960); TschorSNig et al. (2004); HuBENOv (2008a).

\section{ESTHERIA Robineau-Desvoidy, 1830}

Estheria petiolata (Bonsdorff, 1866)

Literature: LANGHOFfer (1920); BARANov (1928); Tschorsnig et al. (2004); Hubenov

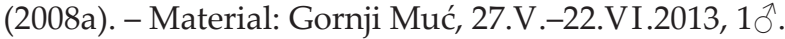

Estheria picta (Meigen, 1826)

Literature: Lutovinovas et al. (2018).

\section{PROSENA Le Peletier \& Serville, 1828}

Prosena siberita (Fabricius, 1775) [= Prosena sybarita Rondani, 1861]

Literature: Strobl (1893); Thalhammer (1899); LANGHoffer (1920); BARANov (1928); Tschorsnig et al. (2004); Hubenov (2008a).

\section{TRIXA Meigen, 1824}

Trixa conspersa (Harris, 1776) [= Trixa oestroidea (Robineau-Desvoidy, 1830)]

Literature: Coe (1960); Tschorsnig et al. (2004); Hubenov (2008a).

\section{ZEUXIA Meigen, 1826}

Zeuxia cinerea Meigen, 1826

Literature: Strobl (1893); Thalhammer (1899); LANGHoffer (1920); BARANov (1928);

Tschorsnig et al. (2004); Hubenov (2008a). - Material: Senj, 18.V.2007, $20 \hat{\jmath}$.

Zeuxia erythraea (Egger, 1856)

Literature: LANGHOFFer (1920); BARANOv (1928); Herting (1984); Tschorsnig et al. (2004); Tschorsnig \& Herting (2005); Hubenov (2008a). - Material: Gornji Muć, 27.V.-13.VII.2013, 2 ㅇ․

Zeuxia subapennina Rondani, 1862

Literature: Tschorsnig et al. (2004); Tschorsnig (2005; 2017); Hubenov (2008a).

Zeuxia tessellata Egger, 1860

Literature: Strobl (1893; 1902; 1904); Thalhammer (1899); LANGHoffer (1920); BAranov (1928); Herting, 1984; Tschorsnig et al. (2004); Tschorsnig \& Herting (2005a); Hubenov (2008a). - Material: Gornji Muć, 27.V.-28.VII.2013, 1§̂, 10 우; 24.V.-20.VII.2014, 7 우. 
Dufouriini Robineau-Desvoidy, 1830

DUFOURIA Robineau-Desvoidy, 1830

Dufouria nigrita (Fallén, 1810)

Literature: Tschorsnig et al. (2004); Hubenov (2008a). - Material: Gornji Muć, 27.V.22.VI.2013, 1万, 4우; 08.-28.VI.2014, 2 우․

\section{MICROSOMA Macquart, 1855}

Microsoma exiguum (Meigen, 1824) [= Syntomogaster singularis Egger, 1860]

Literature: Strobl (1902; 1904); Tschorsnig et al. (2004); Hubenov (2008a). - Material: Gornji Muć, 27.V.-15.IX.2013, 10, 2우; 14.-28.IX.2014, 1 우.

RONDANIA Robineau-Desvoidy, 1850

Rondania cucullata Robineau-Desvoidy, 1850

Literature: Tschorsnig et al. (2004); Hubenov (2008a).

Rondania dimidiata (Meigen, 1824)

Literature: Tschorsnig et al. (2004); Hubenov (2008a). - Material: Gornji Muć, 16. III.-11.IV.2014, 1 ㅇ.

Rondania dispar (Dufour, 1851) [= Hyalomyia dispar Dufour, 1851; = Xysta lesinensis Strobl, 1893]

Literature: Strobl (1893; 1900); Herting (1984); Britvec (2004); Tschorsnig et al. (2004); Hubenov (2008a).

Eutherini Loew, 1854

\section{EUTHERA Loew, 1854}

Euthera fascipennis (Loew, 1854) [= Euthera mannii Mik, 1889]

Literature: Strobl (1898; 1900); Herting (1984); Tschorsnig et al. (2004); Hubenov (2008a).

Voriini Townsend, 1912

ATHRYCIA Robineau-Desvoidy, 1830

Athrycia trepida (Meigen, 1824)

Literature: LANGHoffer (1920); BArAnov (1928); Tschorsnig et al. (2004); Hubenov (2008a).

BLEPHAROMYIA Brauer \& Bergenstamm, 1889

Blepharomyia pagana (Meigen, 1824)

Literature: Lutovinovas et al. (2018).

\section{CAMPYLOCHETA Rondani, 1859}

Campylocheta confusa Ziegler, 1996

Literature: Ziegler (1996); Tschorsnig et al. (2004); Hubenov (2008a). - Material: Gornji Muć, 16.III.-27.IV.2014, 1§, 2 우오.

Campylocheta inepta (Meigen, 1824)

Literature: Lutovinovas et al. (2018).

Campylocheta latigena Mesnil, 1974

Literature: Lutovinovas et al. (2018). 


\section{CYRTOPHLEBA Rondani, 1856}

Cyrtophleba ruricola (Meigen, 1824) [= Plagia ruricola Meigen, 1824]

Literature: Frauenfeld (1860); Tschorsnig et al. (2004); Hubenov (2008a). - Material: Gornji Muć, 27.V.-11.VIII.2013, 2ðð

\section{ERIOTHRIX Meigen, 1803}

Eriothrix apennina (Rondani, 1862) [= Olivieria latifrons Brauer, 1898]

Literature: Strobl (1902; 1904); Coe (1960); Herting (1984); Tschorsnig et al. (2004); Hubenov (2008a).

Eriothrix prolixa (Meigen, 1824)

Literature: Tschorsnig et al. (2004); Hubenov (2008a).

Eriothrix rufomaculata (De Geer, 1776)

Literature: LANGHOFFer (1920); BARANov (1928); Tschorsnig et al. (2004); Hubenov (2008a). - Material: Gornji Muć, 11.VIII.-15.IX.2013, 1§ో; 24.VIII.-14.IX.2014, $1 \widehat{0}, 1$.

\section{PERISCEPSIA Gistel, 1848}

Periscepsia carbonaria (Panzer, 1798) [= Scopolia carbonaria Panzer, 1798]

Literature: Strobl (1893); Baranov (1928). - Material: Gornji Muć, 11.VIII.-06.X.2013, 1; 24.VIII.-14.IX.2014, 1; Omiš, 08.IX.2013, 1 ㅇ.

\section{PHYLLOMYA Robineau-Desvoidy, 1830}

Phyllomya volvulus (Fabricius, 1794) [= Melanota volvolus Fabricius, 1794]

Literature: Thalhammer (1899); LANGHOFFer (1920); BARANov (1928); Tschorsnig et al. (2004); Hubenov (2008a).

\section{RAMONDA Robineau-Desvoidy, 1863}

Ramonda plorans (Rondani, 1861) [= Petinops schnablii Brauer \& Bergenstamm, 1891]

Literature: Brauer \& Bergenstamm (1891); Herting (1984); Tschorsnig et al. (2004); Hubenov (2008a).

Ramonda prunaria (Rondani, 1861)

Literature: Tschorsnig et al. (2004); Hubenov (2008a). - Material: Gornji Muć, 22.

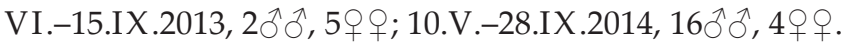

Ramonda prunicia (Herting, 1969)

Literature: Tschorsnig et al. (2004); Hubenov (2008a).

Ramonda spathulata (Fallén, 1820)

Literature: Tschorsnig et al. (2004); Hubenov (2008a). - Material: Gornji Muć, 16.

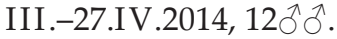

\section{STOMINA Robineau-Desvoidy, 1830}

Stomina caliendrata (Rondani, 1862)

Literature: Tschorsnig et al. (2004); Hubenov (2008a). - Material: Gornji Muć, 24. VIII.-28.IX.2014, 2ふふ, 1 ․

Stomina calvescens Herting, 1977

Literature: Tschorsnig et al. (2004); Hubenov (2008a). - Material: Gornji Muć, 29.VI.28.IX.2014, 2 우우. 
Stomina tachinoides (Fallén, 1817)

Literature: Lutovinovas et al. (2018).

THELAIRA Robineau-Desvoidy, 1830

Thelaira nigripes (Fabricius, 1794)

Literature: LANghoffer (1920); Baranov (1928); Tschorsnig et al. (2004); Hubenov (2008a). - Material: Gornji Muć, 16.III.-11.IV.2014, 1ठ․

Thelaira solivaga (Harris, 1780)

Literature: Tschorsnig et al. (2004); Hubenov (2008a).

VORIA Robineau-Desvoidy, 1830

Voria ruralis (Fallén, 1810)

Literature: LANGHoffer (1920); Baranov (1928); MACElJSKi \& BAlARIN (1975); RuCNER (1994); Britvec (2000; 2004); TsChorsnig et al. (2004); Hubenov (2008a). -

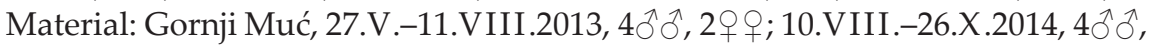
90 ㅇ.

\section{WAGNERIA Robineau-Desvoidy, 1830}

Wagneria cunctans (Meigen, 1824)

Literature: LANGHOFFer (1920). - Material: Gornji Muć, 16.III.-11.IV.2014, $2 \widehat{\jmath}$.

Exoristinae Robineau-Desvoidy, 1863

Acemyini Brauer \& Bergenstamm, 1889

\section{ACEMYA Robineau-Desvoidy, 1830}

Acemya acuticornis (Meigen, 1824)

Literature: LANGhoffer (1920); BARAnov (1928); Tschorsnig et al. (2004); Hubenov (2008a). - Material: Senj, 18.V.2007, 10.

\section{CERACIA Rondani, 1865}

Ceracia mucronifera Rondani, 1865

Literature: Tschorsnig et al. (2004); Hubenov (2008a). - Material: Gornji Muć, 27.V.06.X.2013, 4仓ึ๐, 18ㅇ; 24.V.-10.X.2014, 9 우.

Blondeliini Robineau-Desvoidy, 1863

\section{BELIDA Robineau-Desvoidy, 1863}

Belida angelicae (Meigen, 1824) [= Lydella angelicae (Meigen, 1824); = Tachina spinuligera (Rondani, 1861)]

Literature: Strobl (1893); Thalhammer (1899); Langhoffer (1920); Baranov (1928); Coe (1962); Britvec (2000; 2004); Tschorsnig et al. (2004); Hubenov (2008a).

\section{BLONDELIA Robineau-Desvoidy, 1830}

Blondelia nigripes (Fallén, 1810) [= Lydella nigripes (Fallén, 1810)]

Literature: Kovačević (1926; 1928); Burgess \& Crossman (1929); Dowden (1933); Britvec (2004); Herting (2017); Tschorsnig (2017); Lutovinovas et al. (2018). 


\section{COMPSILURA Bouché, 1834}

Compsilura concinnata (Meigen, 1824) [= Machaira serriventris (Rondani, 1859); = Phorocera taeniata (Meigen, 1824)]

Literature: Strobl (1893; 1902; 1904); Thalhammer (1899); LANGHOFFer (1920); BAranov (1928); Kovačević (1928); Androić (1956; 1957); Rubin (2000); Britvec (2000; 2004); Tschorsnig et al. (2004); Hubenov (2008a); Robertson \& Shaw

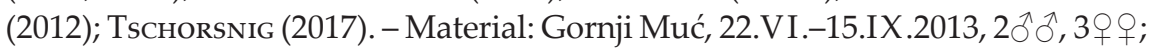
21.VII.-28.IX.2014, 3 우.

\section{CONOGASTER Brauer \& Bergenstamm, 1891}

Conogaster pruinosa (Meigen, 1824)

Literature: Tschorsnig et al. (2004); Tschorsnig \& Herting (2005); Hubenov (2008a).

\section{GASTROLEPTA Rondani, 1862}

Gastrolepta anthracina (Meigen, 1826)

Literature: Frauenfeld (1856). - Material: Gornji Muć, 10.-24.VIII.2014, 1 ․

\section{ISTOCHETA Rondani, 1859}

Istocheta subcinerea (Borisova-Zinoveva, 1966)

Literature: Lutovinovas et al. (2018).

Istocheta sublutescens Herting, 1975

Literature: Herting (1984); Tschorsnig et al. (2004); Hubenov (2008a).

\section{LIGERIA Robineau-Desvoidy, 1863}

Ligeria angusticornis (Loew, 1847)

Literature: Lutovinovas et al. (2018).

\section{LIGERIELLA Mesnil, 1961}

Ligeriella aristata (Villeneuve, 1911)

Literature: Lutovinovas et al. (2018).

\section{LOMACHANTHA Rondani, 1859}

Lomachantha parra Rondani, 1859

Literature: Lutovinovas et al. (2018).

MEDINA Robineau-Desvoidy, 1830

Medina collaris (Fallén, 1820)

Literature: Lutovinovas et al. (2018).

Medina melania (Meigen, 1824)

Literature: Thalhammer (1899); Strobl (1902; 1904). - Material: Gornji Muć, 27.V.22.VI.2013, 4 우; 27.IV.-28.VI.2014, 2 우.

\section{MEIGENIA Robineau-Desvoidy, 1830}

Meigenia dorsalis (Meigen, 1824)

Literature: Tschorsnig et al. (2004); Hubenov (2008a).

Meigenia majuscula (Rondani, 1859)

Literature: LANGHOFFer (1920); BARANov (1928); Tschorsnig et al. (2004); Hubenov (2008a). - Material: Gornji Muć, 11.-27.IV.2014, 1ð̄, 1 ㅇ․ 
Meigenia mutabilis (Fallén, 1810) [= Meigenia bisignata (Meigen, 1824)]

Literature: Strobl (1902; 1904); Langhoffer (1920); Baranov (1928); Coe (1962);

Tschorsnig et al. (2004); Hubenov (2008a). - Material: Gornji Muć, 27.V.-11.

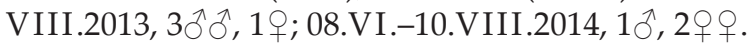

Meigenia simplex Tschorsnig \& Herting, 1998

Literature: Lutovinovas et al. (2018).

OSWALDIA Robineau-Desvoidy, 1863

Oswaldia muscaria (Fallén, 1810)

Literature: Strobl (1902; 1904); Tschorsnig et al. (2004); Hubenov (2008a).

Oswaldia spectabilis (Meigen, 1824)

Literature: Lutovinovas et al. (2018).

\section{PICCONIA Robineau-Desvoidy, 1863}

Picconia incurva (Zetterstedt, 1844) [= Thryptocera laticornis auct. nec (Meigen, 1822)]

Literature: Strobl (1902; 1904); LANGHoffer (1920); Baranov (1928); Mesnil (19441975); Herting (1984); Cooper \& O'Hara (1996); Tschorsnig et al. (2004); HubeNov (2008a). - Material: Gornji Muć, 27.V.-22.VI.2013, 1 ㅇ.

\section{ZAIRA Robineau-Desvoidy, 1830}

Zaira cinerea (Fallén, 1810) [= Viviania cinerea (Fallén, 1810); = Viviania pacta (Meigen, 1824)]

Literature: Brauer \& Bergenstamm (1894); Strobl (1898; 1900); Bjegović (1957); Tschorsnig et al. (2004); Hubenov (2008a); Tschorsnig (2017). - Material: Gornji Muć, 11.IV.-26.X.2014, 1ð̂, 8 우.

Eryciini Robineau-Desvoidy, 1830

\section{APLOMYA Robineau-Desvoidy, 1830}

Aplomya confinis (Fallén, 1820)

Literature: Strobl (1893; 1898; 1900); Tölg \& FAHringer (1911); LANGHOFFER (1920); Baranov (1928); Tschorsnig et al. (2004); Hubenov (2008a). - Material: Gornji

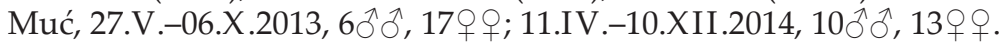

\section{BACTROMYIA Brauer \& Bergenstamm, 1891}

Bactromyia aurulenta (Meigen, 1824)

Literature: Pernek et al. (2016); Tschorsnig (2017).

\section{BUQUETIA Robineau-Desvoidy, 1847}

Buquetia musca Robineau-Desvoidy, 1847

Literature: Tschorsnig et al. (2004); Hubenov (2008a). - Material: Gornji Muć, 27.V.06.X.2013, 1§, 1; 10.V.-10.X.2014, 1§, 7우.

\section{CARCELIA Robineau-Desvoidy, 1830}

Carcelia bombylans Robineau-Desvoidy, 1830

Literature: Tschorsnig et al. (2004); Hubenov (2008a).

Carcelia dubia (Brauer \& Bergenstamm, 1891)

Literature: Thalhammer (1899); Langhoffer (1920); Baranov (1931); Herting (1984); Tschorsnig et al. (2004); Hubenov (2008a). 
Carcelia falenaria (Rondani, 1859)

Literature: Tschorsnig et al. (2004); Hubenov (2008a). - Material: Gornji Muć, 27.V.-

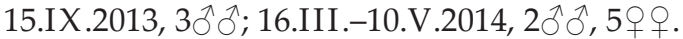

Carcelia gnava (Meigen, 1824) [as Carcelia cheloniae (Rondani, 1859) of KovačEvić (1926), see Herting (2017) and Tschorsnig (2017)]

Literature: LANGHOFFer (1920); KovaČEvić (1926; 1928); BARANOv (1928; 1931); TsChorsnig et al. (2004); Hubenov (2008a); Herting (2017); Tschorsnig (2017).

Carcelia laxifrons Villeneuve, 1912 [as Carcelia cheloniae (Rondani, 1859) of Kovačević (1928), see Tschorsnig (2017)]

Literature: KovačEvić (1928); Tschorsnig (2017).

Carcelia lucorum (Meigen, 1824) [= Carcelia cheloniae (Rondani, 1859); = Exorista cheloniae (Rondani, 1859)]

Literature: Strobl (1893; 1902; 1904); Baranov (1928; 1931); Coe (1960); RuCner (1994); Tschorsnig et al. (2004); Hubenov (2008a).

\section{CATAGONIA Brauer \& Bergenstamm, 1891}

Catagonia aberrans (Rondani, 1859)

Literature: Strobl (1893); Tschorsnig et al. (2004); Hubenov (2008a). - Material: Gornji Muć, 22.VI.-13.VII.2013, 1今̄; 29.VI.-20.VII.2014, 1 ㅇ.

\section{CESTONIA Rondani, 1861}

Cestonia cineraria Rondani, 1861

Literature: Tschorsnig et al. (2004); Hubenov (2008a). - Material: Gornji Muć, 24.VIII.-14.IX.2014, 1 ðै.

\section{CHETINA Rondani, 1856}

Chetina setigena (Rondani, 1856)

Literature: Lutovinovas et al. (2018).

\section{DRINO Robineau-Desvoidy, 1863}

Drino atropivora (Robineau-Desvoidy, 1830) [as tachinid indent. of PAвST (1890a; 1890b) and Novak (1899), see Herting (2017) and Tschorsnig (2017)]

Literature: PAbst (1890a; 1890b); Strobl (1893); Brauer \& Bergenstamm (1894); NoVAK (1899); ThalHAMmer (1899); LANGHOFFer (1920); BARANov (1928); TsChorsNig et al. (2004); Tschorsnig \& Herting (2005); Hubenov (2008a); Herting (2017); TSCHORSNIG (2017).

Drino inconspicua (Meigen, 1830) [= Sturmia cursitans (Rondani, 1861)]

Literature: Strobl (1893); Thalhammer (1899); Burgess \& Crossman (1929); BaranOv (1931); Melis (1940); Tschorsnig et al. (2004); Hubenov (2008a); Tschorsnig (2017). - Material: Gornji Muć, 22.VI.-28.VII.2013, 2 우.

EPICAMPOCERA Macquart, 1849

Epicampocera succincta (Meigen, 1824)

Literature: Langhoffer (1920); Baranov (1928). - Material: Gornji Muć, 28.IX.10.X.2014, 1 ㅇ.

\section{ERYCIA Robineau-Desvoidy, 1830}

Erycia fasciata Villeneuve, 1924

Literature: Herting (1984); Mihályi (1986); Tschorsnig et al. (2004); Hubenov (2008a); TsCHORSNIG (2017). 


\section{LYDELLA Robineau-Desvoidy, 1830}

Lydella grisescens Robineau-Desvoidy, 1830

Literature: Lutovinovas et al. (2018).

Lydella thompsoni Herting, 1959 [= Ceromasia juvenilis auct. nec (Rondani, 1861); = Ceromasia senilis auct. nec (Meigen, 1928); as Lydella grisescens Robineau-Desvoidy, 1830 of Hadžistević (1961), see Herting (2017) and Tschorsnig (2017)]

Literature: Baranov \& Hergula (1928); Hergula (1928; 1929); Baranov (1930c); Hadžistević (1961); Britvec (2000; 2004); Tschorsnig et al. (2004); Hubenov (2008a); Herting (2017); Tschorsnig (2017).

\section{PHEBELLIA Robineau-Desvoidy, 1846}

Phebellia nigripalpis (Robineau-Desvoidy, 1847) [= Parexorista setosa Brauer \& Bergenstamm 1891]

Literature: Brauer \& Bergenstamm (1891); Thalhammer (1899); Herting (1984); Tschorsnig et al. (2004); Hubenov (2008a).

PHRYXE Robineau-Desvoidy, 1830

Phryxe caudata (Rondani, 1859)

Literature: Herting (1993); Rubin (2000); Tschorsnig et al. (2004); Hubenov (2008a); TsCHORSNIG (2017).

Phryxe magnicornis (Zetterstedt, 1838)

Literature: Lutovinovas et al. (2018).

Phryxe nemea (Meigen, 1824)

Literature: Kovačević (1926; 1928); Pernek et al. (2016); Herting (2017); Tschorsnig (2017). - Material: Gornji Muć, 11.IV.-28.VI.2014, $2 \widehat{\jmath}$ ð.

Phryxe prima (Brauer \& Bergenstamm, 1889)

Literature: Strobl (1893); Thalhammer (1899); Tschorsnig et al. (2004); Tschorsnig \& Herting (2005); Hubenov (2008a); Tschorsnig (2017).

Phryxe vulgaris (Fallén, 1810) [= Tachina vernalis Robineau-Desvoidy, 1863]

Literature: Strobl (1893; 1898; 1900; 1902; 1904); ThalHAmmer (1899); LANGHoffer (1920); KovačEvić (1926; 1928); Baranov (1928); Britvec (2000; 2004); TschorSnig et al. (2004); Hubenov (2008a); Herting (2017); Tschorsnig (2017). - Material: Gornji Muć, 27.V.-22.VI.2013, 1; 10.-24.V.2014, 1 ㅇ․

\section{PSEUDOPERICHAETA Brauer \& Bergenstamm, 1899}

Pseudoperichaeta nigrolineata (Walker, 1853) [= Zenillia roseanae (Brauer \& Bergenstamm, 1891)]

Literature: Baranov (1929c); Hergula (1929); Parker et al. (1929); Britvec (2004); Tschorsnig et al. (2004); Hubenov (2008a); Herting (2017); Tschorsnig (2017).

Pseudoperichaeta palesioidea (Robineau-Desvoidy, 1830)

Literature: Tschorsnig et al. (2004); Hubenov (2008a). - Material: Gornji Muć, 29. VI.-20.VII.2014, 1 ㅇ.

\section{PTESIOMYIA Brauer \& Bergenstamm, 1893}

Ptesiomyia alacris (Meigen, 1824)

Literature: Tschorsnig et al. (2004); Hubenov (2008a).

\section{SENOMETOPIA Macquart, 1834}

Senometopia confundens (Rondani, 1859) [= Parexorista flavicans auct. nec (Macquart)]

Literature: Thalhammer (1899); Baranov (1931); Tschorsnig et al. (2004); Hubenov (2008a). 
Senometopia excisa (Fallén, 1820)

Literature: Strobl (1893; 1898; 1900; 1902; 1904); Thalhammer (1899); LANGHoffer (1920); Baranov (1928; 1931); Coe (1962); Tschorsnig et al. (2004); Hubenov (2008a).

Senometopia separata (Rondani, 1859) [as Carcelia cheloniae (Rondani, 1859) of KovačEvić (1926), see Herting (2017) and Tschorsnig (2017)]

Literature: Kovačević (1926; 1928); Herting (2017); Tschorsnig (2017).

Senometopia susurrans (Rondani, 1859)

Literature: BARANOv (1931); Tschorsnig et al. (2004); HubENov (2008a).

Senometopia sp. [as Pales pavida (Meigen, 1824) of Pernek et al. (2013), see Tschorsnig (2017)]

Literature: PERnek et al. (2013; 2016); Tschorsnig (2017).

\section{THECOCARCELIA Townsend, 1933}

Thecocarcelia acutangulata (Macquart, 1850)

Literature: Lutovinovas et al. (2018).

\section{TOWNSENDIELLOMYIA Baranov, 1932}

Townsendiellomyia nidicola (Townsend, 1908) [prb. erroneous of KovačEvić (1926), see Herting (2017) and Tschorsnig (2017)]

Literature: Kovačević (1926; 1928); Tschorsnig et al. (2004); Hubenov (2008a); HerTING (2017); TsCHORSNIG (2017).

Ethillini Mesnil, 1944

\section{ATYLOMYIA Brauer, 1898}

Atylomyia loewii Brauer, 1898

Literature: Lutovinovas et al. (2018).

\section{ETHILLA Robineau-Desvoidy, 1863}

Ethilla aemula (Meigen, 1824)

Literature: Tschorsnig et al. (2004); Hubenov (2008a).

\section{PARATRYPHERA Brauer \& Bergenstamm, 1891}

Paratryphera barbatula (Rondani, 1859)

Literature: Lutovinovas et al. (2018).

Paratryphera bisetosa (Brauer \& Bergenstamm, 1891)

Literature: Lutovinovas et al. (2018).

Exoristini Robineau-Desvoidy, 1863

BESSA Robineau-Desvoidy, 1830

Bessa parallela (Meigen, 1824)

Literature: Tschorsnig et al. (2004); Tschorsnig \& Herting (2005); Hubenov (2008a);

TsCHORSNig (2017).

\section{CHETOGENA Rondani, 1856}

Chetogena acuminata Rondani, 1859

Literature: Ронјоіммӓкі et al. (2016). - Material: Gornji Muć, 11.VIII.-15.IX.2013, 1 . Chetogena filipalpis Rondani, 1859

Literature: Strobl (1893); Thalhammer (1899); LANGHoffer (1920); BARANov (1928); Tschorsnig et al. (2004); Hubenov (2008a). - Material: Gornji Muć, 27.V.-22. VI.2013, $1 \delta^{\lambda}$. 
Chetogena micronychia (Masson, 1969)

Literature: Lutovinovas et al. (2018).

Chetogena obliquata (Fallén, 1810)

Literature: TöLg \& FAHringer (1911); LANGHOFFer (1920); TsChorsnig et al. (2004); Hubenov (2008a).

Chetogena rondaniana (Villeneuve, 1931)

Literature: Herting (1984); Tschorsnig et al. (2004); Hubenov (2008a).

\section{DIPLOSTICHUS Brauer \& Bergenstamm, 1889}

Diplostichus janitrix (Hartig, 1838)

Literature: Tschorsnig et al. (2004); Hubenov (2008a); Tschorsnig (2017).

\section{EXORISTA Meigen, 1803}

Exorista civilis (Rondani, 1859) [= Eutachina civilis (Rondani, 1859)]

Literature: BARAnov (1930b; 1932); Britvec (2004); Herting (2017); Tschorsnig (2017).

Exorista deligata Pandellé, 1896 [= Micropalpus aberrans Strobl, 1893; = Tachina aberrans (Strobl, 1893)]

Literature: Strobl (1893; 1898; 1900; 1902; 1904); Baranov (1928); Coe (1960); Herting (1984; 1985); BRitvec (2000; 2004); Tschorsnig et al. (2004); Hubenov (2008a); TsCHORSNIG (2017).

Exorista florentina Herting, 1975

Literature: Herting (1975); Tschorsnig \& Herting (1994).

Exorista glossatorum (Rondani, 1959)

Literature: Tschorsnig et al. (2004); Hubenov (2008a).

Exorista grandis (Zetterstedt, 1844)

Literature: Tschorsnig et al. (2004); Hubenov (2008a); Tschorsnig (2017).

Exorista larvarum (Linnaeus, 1758)

Literature: Thalhammer (1899); Langhoffer (1920); Kovačević (1928); Melis (1940); Britvec (2004); Tschorsnig et al. (2004); Hubenov (2008a); Tschorsnig (2017).

Exorista nympharum (Rondani, 1859)

Literature: Thalhammer (1899); Tschorsnig et al. (2004); Hubenov (2008a).

Exorista rustica (Fallén, 1810)

Literature: Baranov (1928); Tschorsnig et al. (2004); Hubenov (2008a).

Exorista segregata (Rondani, 1859) [= Parasetigena segregata (Rondani, 1859)]

Literature: Langhoffer (1920); Melis (1940); Tschorsnig et al. (2004); Hubenov (2008a); TsCHORSNIG (2017).

Exorista unicolor (Stein, 1924)

Literature: Herting (1984); Tschorsnig et al. (2004); Hubenov (2008a). - Material: Gornji Muć, 22.VI.-13.VII.2013, 1 ㅇ.

Exorista xanthaspis (Wiedemann, 1830)

Literature: Baranov (1930b); Tschorsnig et al. (2004); Hubenov (2008a).

\section{PHORINIA Robineau-Desvoidy, 1830}

Phorinia aurifrons Robineau-Desvoidy, 1830

Literature: Tschorsnig et al. (2004); Hubenov (2008a). - Material: Gornji Muć, 28. VII.-11.VIII.2013, 1우 10.-24.V.2014, 1 ㅇ․ 


\section{PHOROCERA Brauer \& Bergenstamm, 1889}

Phorocera assimilis (Fallén, 1810) [= Phorocera agilis Robineau-Desvoidy, 1930]

Literature: LANGHOFFER (1920); BARANOv (1928); BRITVEC (2000; 2004); TsCHORSNig et al. (2004); Hubenov (2008a).

Phorocera obscura (Fallén, 1810) [= Phorocera caesifrons Macquart, 1850]

Literature: BArAnov (1928); Britvec (2000; 2004); Tschorsnig et al. (2004); Hubenov (2008a).

Goniini Lioy, 1864

\section{BAUMHAUERIA Meigen, 1838}

Baumhaueria microps Mesnil, 1963

Literature: Tschorsnig et al. (2004); Hubenov (2008a). - Material: Gornji Muć, 16. III.-27.IV.2014, 1§̄, 1 ㅇ.

\section{BLEPHARIPA Rondani, 1856}

Blepharipa pratensis (Meigen, 1824) [= Masicera pratensis (Meigen, 1824); = Crossocosmia scutellata (Robineau-Desvoidy, 1830)]

Literature: Strobl (1893); Thalhammer (1899); Baranov (1928); Coe (1962); Britvec (2000; 2004); Tschorsnig et al. (2004); Hubenov (2008a).

\section{BOTRIA Rondani, 1856}

Botria frontosa (Meigen, 1824) [= Bothria pascuorum Rondani, 1859]

Literature: BARANov (1928); Tschorsnig et al. (2004); HubENov (2008a).

\section{BRACHICHETA Rondani, 1861}

Brachicheta strigata (Meigen, 1824)

Literature: Lutovinovas et al. (2018).

\section{CADURCIA Villeneuve, 1926}

Cadurcia casta (Rondani, 1861)

Literature: LuTOvinovas et al. (2018).

\section{CERATOCHAETOPS Mesnil, 1954}

Ceratochaetops delphinensis (Villeneuve, 1931)

Literature: Lutovinovas et al. (2018).

\section{CEROMASIA Rondani, 1856}

Ceromasia rubrifrons (Macquart, 1834) [= Ceromasia florum (Macquart, 1850)]

Literature: Thalhammer (1899); Tschorsnig et al. (2004); Hubenov (2008a).

\section{CLEMELIS Robineau-Desvoidy, 1863}

Clemelis pullata (Meigen, 1824) [= Phorocera polleniella Rondani, 1859]

Literature: Strobl (1893; 1898; 1900); Thalhammer (1899); HAdžIstević (1961); TsCHORSNig et al. (2004); Hubenov (2008a); Tschorsnig (2017). - Material: Gornji Muć, 22.VI.-06.X.2013, 3q + ; 29.VI.-28.IX.2014, 4 $q$.

\section{CYZENIS Robineau-Desvoidy, 1863}

Cyzenis albicans (Fallén, 1810)

Literature: Tschorsnig et al. (2004); Hubenov (2008a). 


\section{DOLICHOCOLON Brauer \& Bergenstamm, 1889}

Dolichocolon paradoxum Brauer \& Bergenstamm, 1889

Literature: Brauer \& Bergenstamm (1889); Strobl (1893; 1898); Herting (1974; 1984); Hubenov (2008a); Cerretti \& Shima (2011); O’Hara \& Cerretti (2016). - Material: Gornji Muć, 27.V.-22.VI.2013, 1q; 21.VII.-10.VIII.2014, 1 ㅇ.

ERYNNIA Robineau-Desvoidy, 1830

Erynnia ocypterata (Fallén, 1810) [= Erynnia nitida Robineau-Desvoidy, 1830]

Literature: Strobl (1893; 1898; 1900); Thalhammer (1899); Tschorsnig et al. (2004); Hubenov (2008a).

\section{ERYTHROCERA Robineau-Desvoidy, 1848}

Erythrocera nigripes (Robineau-Desvoidy, 1830) [= Pexomya rubifrons (Perris, 1852)]

Literature: LANghoffer (1920); BArAnov (1928); Tschorsnig et al. (2004); Hubenov (2008a).

\section{EUMEA Robineau-Desvoidy, 1863}

Eumea linearicornis (Zetterstedt, 1844)

Literature: Lutovinovas et al. (2018).

\section{EURYSTHAEA Robineau-Desvoidy, 1863}

Eurysthaea scutellaris (Robineau-Desvoidy, 1848)

Literature: WACHTL (1889); BARANOv (1928); Tschorsnig et al. (2004); TsCHORSNIG \& Herting (2005); Hubenov (2008a); Pernek et al. (2016); Tschorsnig (2017).

\section{FRONTINA Meigen, 1838}

Frontina laeta (Meigen, 1824)

Literature: Frauenfeld (1856); LANGHOFFER (1920); BARANOv (1928); Tschorsnig et al. (2004); Hubenov (2008a).

\section{GONIA Meigen, 1803}

Gonia bimaculata Wiedemann, 1819

Literature: Tschorsnig et al. (2004); Hubenov (2008a).

Gonia capitata (De Geer, 1776)

Literature: Frauenfeld (1856); LANGHOFFER (1920); BARANOv (1928); Tschorsnig et al. (2004); Hubenov (2008a).

Gonia divisa Meigen, 1826

Literature: LANGHoffer (1920); BARAnov (1928); Tschorsnig et al. (2004); Hubenov (2008a).

Gonia ornata Meigen, 1826 [= Gonia lateralis Zeller, 1842]

Literature: Strobl (1902; 1904); TöLg \& FAHringer (1911); Tschorsnig et al. (2004); Hubenov (2008a).

Gonia picea (Robineau-Desvoidy, 1830) [= Gonia fasciata Meigen, 1826]

Literature: LANghoffer (1920); Tschorsnig et al. (2004); Hubenov (2008a). - Material: Gornji Muć, 16.III.-11.IV.2014, 1 ․

Gonia vacua Meigen, 1826

Literature: Tschorsnig et al. (2004); HubEnov (2008a). 


\section{MASICERA Macquart, 1834}

Masicera pavoniae (Robineau-Desvoidy, 1830) [as Tachina silvatica Fallén, 1810 of BrisChKe (1885) and Riedel (1908), and tachinids indent. of Kuhlmann (1890), see Herting (2017) and Tschorsnig (2017)]

Literature: Brischke (1885); Kuhlmann (1890); Riedel (1908); Herting (1960; 2017); Tschorsnig et al. (2004); Tschorsnig \& Herting (2005); Mückstein et al. (2007); Hubenov (2008a); Tschorsnig (2017).

Masicera silvatica (Fallén, 1810) [= Tachina silvatica Fallén, 1810]

Literature: Strobl (1902; 1904); LAnghoffer (1920); Baranov (1928); Tschorsnig (1990); Tschorsnig et al. (2004); Hubenov (2008a).

Masicera sphingivora (Robineau-Desvoidy, 1830) [as Masicera silvatica (Fallén, 1810) and Masicera pratensis (Meigen, 1824) of Kovačević (1928), see Herting (2017) and Tschorsnig (2017)]

Literature: Kovačević (1928); Tschorsnig et al. (2004); Tschorsnig (2005; 2017); HuBenov (2008a); Herting (2017). - Material: Gornji Muć, 11.VIII.-06.X.2013, 1q; 10.V.-10.VIII.2014, 2 우.

\section{OCYTATA Gistel, 1848}

Ocytata pallipes (Fallén, 1820)

Literature: RuCNER (1994). - Material: Gornji Muć, 27.V.-22.VI.2013, 19; 24.VIII.23.XI.2014, $2 \widehat{\diamond}, 1$ ㅇ.

\section{PALES Robineau-Desvoidy, 1830}

Pales pavida (Meigen, 1824) [= Phorocera cilipeda Rondani, 1895]

Literature: Strobl (1900); Cerretti (2005); Hubenov (2008a); Pernek et al. (2016); TsCHORSNig (2017). - Material: Gornji Muć, 10.VIII.-23.XI.2014, 4우; Molunat, 21.V.2007, $10^{\uparrow}$.

Pales pumicata (Meigen, 1824)

Literature: Lutovinovas et al. (2018).

\section{PEXOPSIS Meigen, 1824}

Pexopsis aprica (Meigen, 1824)

Literature: Tschorsnig et al. (2004); Hubenov (2008a).

\section{PHRYNO Robineau-Desvoidy, 1830}

Phryno vetula (Meigen, 1824)

Literature: Lutovinovas et al. (2018).

\section{PLATYMYA Robineau-Desvoidy, 1830}

Platymya antennata (Brauer \& Bergenstamm, 1891)

Literature: Tschorsnig et al. (2004); Hubenov (2008a).

Platymya fimbriata (Meigen, 1824)

Literature: Lutovinovas et al. (2018).

\section{PROSOPEA Rondani, 1861}

Prosopea nigricans (Egger, 1861)

Literature: Thalhammer (1899); Tschorsnig et al. (2004); Hubenov (2008a). - Material: Gornji Muć, 08.VI.-20.VII.2014, 4 우. 


\section{PSEUDOGONIA Brauer \& Bergenstamm, 1889}

Pseudogonia parisiaca (Robineau-Desvoidy, 1851)

Literature: Tschorsnig et al. (2004); Hubenov (2008a).

Pseudogonia rufifrons (Wiedemann, 1830)

Literature: Tschorsnig et al. (2004); Hubenov (2008a). - Material: Gornji Muć, 27.V .22.VI.2013, 1 ㅇ․

\section{SPALLANZANIA Robineau-Desvoidy, 1830}

Spallanzania hebes (Fallén, 1820)

Literature: Strobl (1902; 1904); LANGHOFFER (1920); BARANOv (1928); Tschorsnig et al. (2004); Hubenov (2008a).

Spallanzania rectistylum (Macquart, 1847)

Literature: Herting (1984); Tschorsnig et al. (2004); Hubenov (2008a). - Material:

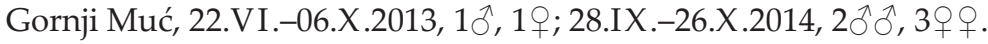

\section{STURMIA Robineau-Desvoidy, 1830}

Sturmia bella (Meigen, 1824) [= Sturmia vanessae Robineau-Desvoidy, 1830]

Literature: LANGHOFFER (1920); BARANOv (1928); BRITVEC (2000; 2004); Tschorsnig et al. (2004); Hubenov (2008a). - Material: Gornji Muć, 24.VIII.-14.IX.2014, 1 q.

\section{ZENILLIA Robineau-Desvoidy, 1830}

Zenillia libatrix (Panzer, 1798) [= Exorista fulva (Fallén, 1810)]

Literature: Frauenfeld (1860); Kovačević (1928); Tschorsnig (2017).

Thrixionini Townsend, 1913

\section{THRIXION Brauer \& Bergenstamm, 1889}

Thrixion aberrans (Schiner, 1862) [as Diptera indent. of Siebold (1843), see Herting (2017) and Tschorsnig (2017)]

Literature: Siebold (1843); Herting (1984; 2017); Tschorsnig et al. (2004); Hubenov (2008a); TsCHORSNIG (2017).

Winthemiini Townsend, 1913

\section{NEMORILLA Rondani, 1856}

Nemorilla floralis (Fallén, 1810)

Literature: Strobl (1893); Thalhammer (1899); Baranov (1928); Britvec (2004); TsCHorsnig et al. (2004); Hubenov (2008a).

Nemorilla maculosa (Meigen, 1824) [as Nemorilla floralis (Fallén, 1810) of Melis (1940), see TschorsNig (2017)]

Literature: Strobl (1893; 1898; 1900); Langhoffer (1920); Melis (1940); Tschorsnig et al. (2004); Hubenov (2008a); Tschorsnig (2017). - Material: Gornji Muć, 11. VIII.-06.X.2013, 1§, 2ㅇ; 10.VIII.-14.IX.2014, 2 우.

\section{SMIDTIA Robineau-Desvoidy, 1830}

Smidtia amoena (Meigen, 1824)

Literature: Lutovinovas et al. (2018). 


\section{WINTHEMIA Robineau-Desvoidy, 1830}

Winthemia quadripustulata (Fabricius, 1794)

Literature: LANGHOFFER (1920); BARANov (1928); TsChORSNig et al. (2004); Hubenov (2008a).

Winthemia venusta (Meigen, 1824)

Literature: Tschorsnig et al. (2004); Hubenov (2008a).

Phasinnae Robineau-Desvoidy, 1830

Catharosiini Townsend, 1936

\section{CATHAROSIA Rondani, 1868}

Catharosia flavicornis (Zetterstedt, 1859)

Literature: Lutovinovas et al. (2018).

Catharosia pygmaea (Fallén, 1815)

Literature: Tschorsnig et al. (2004); Hubenov (2008a). - Material: Gornji Muć, 28.VII.-11.VIII.2013, 1ठ; 11.IV.-10.VIII.2014, 2 우.

Cylindromyiini Townsend, 1912

\section{BESSERIA Robineau-Desvoidy, 1830}

Besseria lateritia (Meigen, 1824)

Literature: Tschorsnig et al. (2004); Hubenov (2008a).

Besseria melanura (Meigen, 1824)

Literature: Strobl (1893; 1902; 1904); Thalhammer (1899); LANGhoffer (1920); BARANOV (1928); TschorsNig et al. (2004); HubenOv (2008a).

Besseria reflexa Robineau-Desvoidy, 1830

Literature: Herting (1984); Tschorsnig et al. (2004); Hubenov (2008a). - Material: Gornji Muć, 27.V.-11.VIII.2013, 2ððત, 10우; Paklenica, 27.V.2002, 1 ㅇ․

Besseria zonaria (Loew, 1847)

Literature: Herting (1984); Tschorsnig et al. (2004); Hubenov (2008a).

\section{CYLINDROMYIA Meigen, 1803}

Cylindromyia auriceps (Meigen, 1838) [= Ocypterula auriceps Meigen, 1838]

Literature: LANGHOFFER (1920); BARANov (1929b); Tschorsnig et al. (2004); Hubenov

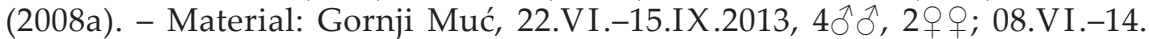
IX.2014, 1ठ, 1; Senj, 18.V.2007, 1ð.

Cylindromyia bicolor (Olivier, 1812)

Literature: LANGHOFFER (1920); BARANOv (1928; 1929b); TsCHORSNIG et al. (2004); Hu-

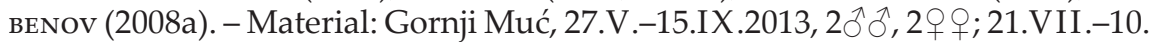
VIII.2014, 1 \% .

Cylindromyia brassicaria (Fabricius, 1775) [= Ocyptera boscii (Robineau-Desvoidy, 1830); = Ocyptera brassicaria Fabricius, 1775]

Literature: Frauenfeld (1860); Strobl (1902; 1904); LANGHoffer (1920); Baranov (1928; 1929b); Tschorsnig et al. (2004); Hubenov (2008a). - Material: Gornji Muć, 27.V.-15.IX.2013, 7ふふえ, 6우; 08.VI.-24.VIII.2014, 2ふふえ, 2 우. 
Cylindromyia crassa (Loew, 1845)

Literature: Lutovinovas et al. (2018).

Cylindromyia intermedia (Meigen, 1824) [= Cylindromyia excisa (Loew, 1845); = Ocyptera intermedia Meigen, 1824; = Ocyptera scalaris Loew, 1844]

Literature: STRobl (1893; 1904); ThalHammer (1899); LANGHOFFER (1920); BARANov (1928; 1929b); Coe (1960); Tschorsnig et al. (2004); Hubenov (2008a). - Material:

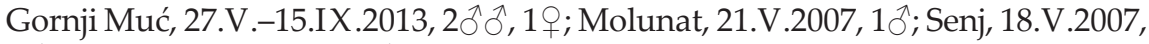
$1{ }^{\lambda}$; Vodice, 08.IX.2008, $1 \delta^{\lambda}$.

Cylindromyia interrupta (Meigen, 1824)

Literature: Thalhammer (1899); Strobl (1902; 1904); Langhoffer (1920); Baranov (1928; 1929b); Tschorsnig et al. (2004); Hubenov (2008a).

Cylindromyia pilipes (Loew, 1844) [= Ocyptera pilipes Loew, 1844]

Literature: Strobl (1893; 1902; 1904); LANGHoffer (1896; 1920); ThalHAMmer (1899); Baranov (1928; 1929b); Tschorsnig et al. (2004); Hubenov (2008a).

Cylindromyia pusilla (Meigen, 1824) [= Ocypterula costalis (Loew, 1844); = Ocypterula pusilla Meigen, 1824]

Literature: Strobl (1893; 1898; 1900; 1902; 1904); Thalhammer (1899); LANGHoffer (1920); BarAnov (1928; 1929b); Tschorsnig et al. (2004); Hubenov (2008a).

Cylindromyia rubida (Loew, 1854)

Literature: Loew (1854); Schiner (1862); Strobl (1898; 1900; 1902; 1904); Herting (1984); Tschorsnig et al. (2004); Hubenov (2008a); O’Hara \& Cerretti (2016).

Cylindromyia rufifrons (Loew, 1844)

Literature: Strobl (1893); Thalhammer (1899); LANGhoffer (1920); Baranov (1928); Tschorsnig et al. (2004); Hubenov (2008a). - Material: Gornji Muć, 28.VII.-11. VIII.2013, 1 ㅇ.

Cylindromyia rufipes (Meigen, 1824) [= Ocyptera rufipes Meigen, 1824]

Literature: Frauenfeld (1856); Schiner (1862); Strobl (1893; 1898; 1900); LANGhoffer (1920); Baranov (1928; 1929b); Tschorsnig et al. (2004); Hubenov (2008a).

Cylindromyia xylotina (Egger, 1860)

Literature: Lutovinovas et al. (2018).

\section{HEMYDA Robineau-Desvoidy, 1830}

Hemyda vittata (Meigen, 1824)

Literature: Frauenfeld (1856); Strobl (1898; 1900); Thalhammer (1899); Tschorsnig et al. (2004); Hubenov (2008a).

\section{LOPHOSIA Meigen, 1824}

Lophosia fasciata Meigen, 1824

Literature: BARAnov (1928); Tschorsnig et al. (2004); Hubenov (2008a).

\section{PHANIA Meigen, 1824}

Phania curvicauda (Fallén, 1820)

Literature: Lutovinovas et al. (2018).

Phania funesta (Meigen, 1824) [= Weberia pseudofunesta Villeneuve, 1931]

Literature: Coe (1960); Tschorsnig et al. (2004); Hubenov (2008a). 
Leucostomatini Townsend, 1908

\section{CLAIRVILLIA Robineau-Desvoidy, 1830}

Clairvillia biguttata (Meigen, 1824)

Literature: Strobl (1893; 1902; 1904); Thalhammer (1899); LANGHOFFer (1920); BARANOv (1928); Tschorsnig et al. (2004); HubENOv (2008a).

Clairvillia pninae Kugler, 1971

Literature: Herting (1984); Tschorsnig et al. (2004); Hubenov (2008a).

\section{DIONAEA Robineau-Desvoidy, 1830}

Dionaea aurifrons (Meigen, 1824)

Literature: LANGHOFFer (1920); BARANOv (1928); Tschorsnig et al. (2004); Hubenov (2008a). - Material: Gornji Muć, 22.VI.-28.VII.2013, 1§̂, 1 ㅇ.

\section{EULABIDOGASTER Belanovksy, 1951}

Eulabidogaster setifacies (Rondani, 1861)

Literature: Strobl (1893; 1898; 1900); LANGhoffer (1920); BARANov (1928); TschorSNig et al. (2004); Hubenov (2008a). - Material: Gornji Muć, 27.V.-22.VI.2013, 1; 08.VI.-24.VIII.2014, 1ð, 2 q 9 .

\section{LABIGASTERA Macquart, 1834}

Labigastera nitidula (Meigen, 1824)

Literature: Lutovinovas et al. (2018).

Labigastera pauciseta (Rondani, 1861)

Literature: Lutovinovas et al. (2018).

\section{LEUCOSTOMA Meigen, 1803}

Leucostoma anthracinum (Meigen, 1824)

Literature: Lutovinovas et al. (2018).

Leucostoma crassum Kugler, 1966

Literature: Herting (1984); Tschorsnig et al. (2004); Hubenov (2008a). - Material: Gornji Muć, 27.V.-15.IX.2013, 2 우.

Leucostoma edentatum Kugler, 1978

Literature: Lutovinovas et al. (2018).

Leucostoma simplex (Fallén, 1815) [= Leucostoma aterrima (Villers, 1789)]

Literature: LANGHOFFer (1920); Baranov (1928); Keglević (1970); Tschorsnig et al.

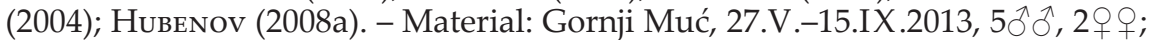
08.VI.-14.IX.2014, 2 ふ઼, 1 ㅇ.

Leucostoma tetraptera (Meigen, 1824)

Literature: Lutovinovas et al. (2018).

Leucostoma turonicum Dupuis, 1964

Literature: Lutovinovas et al. (2018).

Phasiini Robineau-Desvoidy, 1830

\section{CISTOGASTER Latreille, 1804}

Cistogaster globosa (Fabricius, 1775)

Literature: Frauenfeld (1856); Strobl (1893); Thalhammer (1899); Tschorsnig et al. (2004); Hubenov (2008a). - Material: Gornji Muć, 22.VI.-13.VII.2013, 1 ; 29.VI.20.VII.2014, 1今ं; Senj, 18.V.2007, 1 ㅇ. 


\section{CLYTIOMYA Rondani, 1861}

Clytiomya continua (Panzer, 1798) [= Clytia dalmatica Robineau-Desvoidy, 1830]

Literature: Schiner (1862); Strobl (1893; 1898; 1900); Herting (1984); Britvec (2004); Tschorsnig et al. (2004); Hubenov (2008a). - Material: Gornji Muć, 10.-24. VIII.2014, 1 ㅇ.

Clytiomya dupuisi Kugler, 1971

Literature: Tschorsnig et al. (2004); Hubenov (2008a).

Clytiomya mesnili Kugler, 1968

Literature: Lutovinovas et al. (2018).

Clytiomya sola (Rondani, 1861) [= Clytia latifrons Strobl, 1893; = Clytiomyia dalmatica auct. nec (Robineau-Desvoidy, 1830)]

Literature: Strobl (1893); Brauer (1898); Thalhammer (1899); Britvec (2004); TsChorsnig et al. (2004); Hubenov (2008a). - Material: Gornji Muć, 22.VI.-06.X.2013, 2ㅇ; 21.VII.-10.VIII.2014, 1 ․

\section{ECTOPHASIA Townsend, 1912}

Ectophasia crassipennis (Fabricius, 1794) [= Phasia rostrata Egger, 1860; = Phasia strigata Girschner, 1888]

Literature: Strobl (1893; 1902; 1904); ThALHAMmer (1899); LANGHOFFER (1920); BARANOv (1928); Tschorsnig et al. (2004); HubENOv (2008a).

Ectophasia leucoptera (Rondani, 1865)

Literature: Tschorsnig et al. (2004); Hubenov (2008a). - Material: Gornji Muć, 28. IX.-10.X.2014, 1 ㅇ.

Ectophasia oblonga (Robineau-Desvoidy, 1830)

Literature: RIEgER \& TsCHORSNig (2001); TsCHORsNig et al. (2004); TsCHORSNig (2005; 2017); Hubenov (2008a). - Material: Senj, 18.V.2007, 1 ㅇ.

\section{ELIOZETA Rondani, 1856}

Eliozeta helluo (Fabricius, 1805) [= Clytia helvola Meigen, 1824]

Literature: Strobl (1902; 1904); Tschorsnig et al. (2004); Hubenov (2008a).

\section{ELOMYA Robineau-Desvoidy, 1830}

Elomya lateralis (Meigen, 1824)

Literature: Frauenfeld (1860); Strobl (1898); Tschorsnig et al. (2004); Hubenov (2008a).

\section{GYMNOSOMA Meigen, 1803}

Gymnosoma clavatum (Rohdendorf, 1947)

Literature: Tschorsnig et al. (2004); Hubenov (2008a). - Material: Gornji Muć, 27.V.-

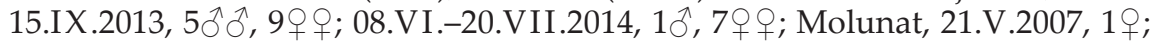
Pula, 07.IX.2009, $1 \delta^{\lambda}$.

Gymnosoma costatum (Panzer, 1800)

Literature: Thalhammer (1899); Strobl (1902; 1904); Herting (1984); Tschorsnig et al. (2004); Hubenov (2008a). - Material: Gornji Muć, 22.VI --28.VII.2013, 1ठ, 1 ㅇ.

\section{Gymnosoma dolycoridis Dupuis, 1961}

Literature: Tschorsnig et al. (2004); Hubenov (2008a). - Material: Gornji Muć, 10.-24. VIII.2014, 1 ․ 
Gymnosoma inornatum Zimin, 1966

Literature: HubEnov (2008a).

Gymnosoma nitens Meigen, 1824

Literature: Strobl (1893; 1898; 1900; 1902; 1904); ThalHAmmer (1899); LANGHOFFer (1920); Baranov (1928); Tschorsnig et al. (2004); Hubenov (2008a). - Material:

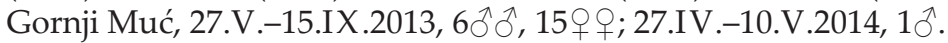

Gymnosoma rotundatum (Linnaeus, 1758)

Literature: Frauenfeld (1856); STROBL (1893; 1898; 1900; 1902; 1904); ThalHAMMER (1899); Langhoffer (1920); Baranov (1928); Tschorsnig et al. (2004); Hubenov (2008a).

Gymnosoma rungsi Mesnil, 1952

Literature: Tschorsnig et al. (2004); HubEnov (2008a).

\section{PHASIA Latreille, 1804}

Phasia aurigera (Egger, 1860) [= Allophora bonapartea (Rondani, 1861)]

Literature: BARANov (1928); Sun \& MARshall (2003); Tschorsnig et al. (2004); HuBENOV (2008a).

Phasia aurulans Meigen, 1824

Literature: Sun \& MARshall (2003).

Phasia hemiptera (Fabricius, 1794) [= Allophora eximia (Girschner, 1887)]

Literature: LANGHoffer (1920); BARANov (1928); Tschorsnig et al. (2004); Hubenov (2008a).

Phasia obesa (Fabricius, 1798)

Literature: Sun \& Marshall (2003); Tschorsnig et al. (2004); Hubenov (2008a).

Phasia pandellei (Dupuis, 1957)

Literature: Herting (1984); Sun \& Marshall (2003); Tschorsnig et al. (2004); HubeNOv (2008a).

Phasia pusilla Meigen, 1824

Literature: Langhoffer (1920); Sun \& MARshall (2003); Tschorsnig et al. (2004); Hubenov (2008a). - Material: Gornji Muć, 24.VIII.-14.IX.2014, $1 \widehat{\jmath}$.

Phasia subcoleoptrata (Linnaeus, 1767)

Literature: Sun \& Marshall (2003); Tschorsnig et al. (2004); Hubenov (2008a).

XYSTA Meigen, 1824

Xysta holosericea (Fabricius, 1805)

Literature: Herting (1984); Tschorsnig et al. (2004); Hubenov (2008a).

Strongygastrini Townsend, 1936

\section{STRONGYGASTER Macquart, 1834}

Strongygaster globula (Meigen, 1824)

Literature: Tschorsnig et al. (2004); Hubenov (2008a).

Trichopodini Townsend, 1908

\section{TRICHOPODA Berthold, 1827}

Trichopoda pennipes (Fabricius, 1781)

Literature: BystrowsKi (2012). 
Tachininae Robineau-Desvoidy, 1830

Bigonichetini Townsend, 1913

TRIARTHRIA Stephens, 1829

Triarthria setipennis (Fallén, 1810)

Literature: Lutovinovas et al. (2018).

Brachymerini Mesnil, 1939

PELAMERA Herting, 1969

Pelamera atra (Rondani, 1861)

Literature: Lutovinovas et al. (2018).

Graphogastrini Townsend, 1931

\section{GRAPHOGASTER Rondani, 1868}

Graphogaster parvipalpis Kugler, 1974

Literature: Tschorsnig et al. (2004); Hubenov (2008a).

Graphogaster vestita Rondani, 1868 [= Paracyrillia maculata Strobl, 1893]

Literature: Strobl (1893; 1898; 1900); Herting (1984); Britvec (2004); Tschorsnig et al. (2004); Hubenov (2008a). - Material: Gornji Muć, 28.VII.-11.VIII.2013, 19; Vir, 28.VI.2011, 1 ㅇ.

PHYTOMYPTERA Rondani, 1845

Phytomyptera nigrina (Meigen, 1824) [= Phytomyptera nitidiventris Rondani, 1845]

Literature: Strobl (1902; 1904); Tschorsnig et al. (2004); Hubenov (2008a).

Ernestiini Townsend, 1912

\section{ELOCERIA Robineau-Desvoidy, 1863}

Eloceria delecta (Meigen, 1824)

Literature: Tschorsnig et al. (2004); Hubenov (2008a).

\section{EURITHIA Robineau-Desvoidy, 1844}

Eurithia anthophila (Robineau-Desvoidy, 1830) [= Ernestia radicum auct. nec (Linnaeus, 1758)]

Literature: LANGHOFfer (1920); BARANov (1928); Tschorsnig et al. (2004); Hubenov (2008a).

Eurithia connivens (Zetterstedt, 1844) [= Ernestia connivens (Zetterstedt, 1844)]

Literature: LANGHOFFer (1920); BARANov (1928); Tschorsnig et al. (2004); Hubenov (2008a).

Eurithia consobrina (Meigen, 1824) [= Ernestia consobrina (Meigen, 1824)]

Literature: LANGHOFFer (1920); BARANov (1928); Tschorsnig et al. (2004); Hubenov (2008a).

\section{LOEWIA Egger, 1856}

Loewia brevifrons (Rondani, 1856)

Literature: Schiner (1862); Strobl (1898; 1900); Herting (1984); Tschorsnig et al. (2004); Hubenov (2008a). 


\section{PANZERIA Robineau-Desvoidy, 1830}

Panzeria rudis (Fallén, 1810) [= Ernestia rudis (Fallén, 1810)]

Literature: LANGHOFFer (1920); BARANOv (1928); Tschorsnig et al. (2004); Hubenov (2008a).

Panzeria vagans (Meigen, 1824) [= Ernestia vagans (Meigen, 1824)]

Literature: Tschorsnig et al. (2004); Hubenov (2008a).

\section{SYNACTIA Villeneuve, 1915}

Synactia parvula (Rondani, 1861)

Literature: Tschorsnig et al. (2004); Hubenov (2008a). - Material: Gornji Muć, 27.V.-

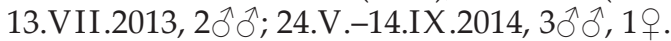

\section{ZOPHOMYIA Macquart, 1835}

Zophomyia temula (Scopoli, 1763)

Literature: LANGHOFFer (1920); BARANOv (1928); Tschorsnig et al. (2004); Hubenov (2008a).

Leskiini Townsend, 1919

\section{APHRIA Robineau-Desvoidy, 1830}

Aphria longirostris (Meigen, 1824)

Literature: Strobl (1893; 1898; 1900); Thalhammer (1899); LANGHoffer (1920); BARANOv (1928); Tschorsnig et al. (2004); Hubenov (2008a). - Material: Gornji Muć, 12.VI.-13.VII.2013, 1 ․

\section{BITHIA Robineau-Desvoidy, 1863}

Bithia acanthophora (Rondani, 1861)

Literature: Tschorsnig et al. (2004); Hubenov (2008a).

Bithia demotica (Egger, 1861)

Literature: Tschorsnig et al. (2004); Hubenov (2008a). Material: Gornji Muć, 27.V.-

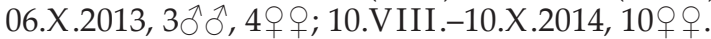

Bithia golanensis (Kugler, 1971)

Literature: Herting (1984); Tschorsnig et al. (2004); Hubenov (2008a).

Bithia immaculata Herting, 1971

Literature: Herting (1984); Tschorsnig et al. (2004); Hubenov (2008a). - Material:

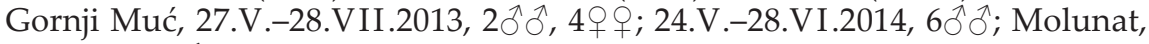
21.V.2007, 1 đ̂.

Bithia proletaria (Egger, 1860)

Literature: Strobl (1893); Thalhammer (1899); LANGHoffer (1920); BARANov (1928); Tschorsnig et al. (2004); Hubenov (2008a).

Bithia spreta (Meigen, 1824) [= Demoticus frontatus (Boheman, 1852)]

Literature: Strobl (1893); Thalhammer (1899); Tschorsnig et al. (2004); Hubenov (2008a).

\section{CLAUSICELLA Rondani, 1856}

Clausicella suturata Rondani, 1859

Literature: Strobl (1893; 1902; 1904); Herting (1984); Tschorsnig et al. (2004); HuBENOV (2008a). 


\section{DEMOTICUS Macquart, 1854}

Demoticus plebejus (Fallén, 1810)

Literature: Thalhammer (1899); Tschorsnig et al. (2004); Hubenov (2008a).

\section{FISCHERIA Robineau-Desvoidy, 1830}

Fischeria bicolor Robineau-Desvoidy, 1830

Literature: Strobl (1893; 1898; 1900; 1902; 1904); Tschorsnig et al. (2004); Hubenov (2008a).

\section{LESKIA Robineau-Desvoidy, 1830}

Leskia aurea (Fallén, 1820) [= Myobia aurea (Fallén, 1820)]

Literature: Frauenfeld (1856); Strobl (1893); Thalhammer (1899); Langhoffer (1920); Baranov (1928); Tschorsnig et al. (2004); Tschorsnig \& Herting (2005); Hubenov (2008a); Tschorsnig (2017). - Material: Gornji Muć, 14.-28.IX.2014, 1 ㅇ․

\section{SOLIERIA Robineau-Desvoidy, 1848}

Solieria fenestrata (Meigen, 1824) [= Myobia fuscana (Robineau-Desvoidy, 1848)]

Literature: LANGHOFFer (1920); BARANov (1928); Tschorsnig et al. (2004); Hubenov (2008a). - Material: Gornji Muć, 27.V.-22.VI.2013, 1 q.

Linnaemyini Townsend, 1919

\section{CHRYSOSOMOPSIS Townsend, 1916}

Chrysosomopsis aurata (Fallén, 1820) [= Chrysocosmius auratus (Fallén, 1820)]

Literature: Thalhammer (1899); Tschorsnig et al. (2004); Hubenov (2008a). - Material: Gornji Muć, 24.VIII.-10.X.2014, 1ð, 2 우.

\section{LINNAEMYA Robineau-Desvoidy, 1830}

\section{Linnaemya comta (Fallén, 1810)}

Literature: BARANov (1928); Coe (1962); Tschorsnig et al. (2004); Hubenov (2008a). - Material: Gornji Muć, 24.V.-08.VI.2014, 1 q .

Linnaemya frater (Rondani, 1859)

Literature: LANGHoffer (1920); BARAnov (1928); Tschorsnig et al. (2004); Hubenov (2008a).

Linnaemya impudica (Rondani, 1859)

Literature: LANGhoffer (1920); BARAnov (1928); Tschorsnig et al. (2004); Hubenov (2008a).

Linnaemya lithosiophaga (Rondani, 1859)

Literature: Strobl (1902; 1904); Tschorsnig et al. (2004); Hubenov (2008a). - Material: Gornji Muć, 21.VII.-28.IX.2014, 10, 8 우을.

Linnaemya soror Zimin, 1954

Literature: Tschorsnig et al. (2004); Hubenov (2008a).

Linnaemya vulpina (Fallén, 1810) [= Micropalpus vulpinus (Fallén, 1810)]

Literature: Frauenfeld (1856); Baranov (1928); Tschorsnig et al. (2004); Hubenov (2008a). - Material: Gornji Muć, 11.VIII.-06.X.2013, 2ふふふ; 24.VIII.-10.X.2014, $6 \widehat{\jmath}, 2$ 우; Vodice, 08.IX.2008, 1 え. 
Macquartiini Robineau-Desvoidy, 1830

\section{MACQUARTIA Robineau-Desvoidy, 1830}

Macquartia chalconota (Meigen, 1824)

Literature: Thalhammer (1899); Tschorsnig et al. (2004); Hubenov (2008a).

Macquartia dispar (Fallén, 1820)

Literature: LANGHOFFER (1920); BARANov (1928); Tschorsnig et al. (2004); Hubenov

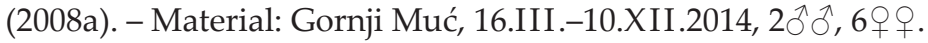

Macquartia tenebricosa (Meigen, 1824) [= Macquartia affinis Schiner, 1862]

Literature: Thalhammer (1899); Tschorsnig et al. (2004); Hubenov (2008a).

Macquartia tessellum (Meigen, 1824)

Literature: Lutovinovas et al. (2018).

Megaprosopini Townsend, 1908

\section{DEXIOSOMA Rondani, 1856}

Dexiosoma caninum (Fabricius, 1781) [= Dexia canina (Fabricius, 1781)]

Literature: Frauenfeld (1860); Langhoffer (1920); Baranov (1928); Tschorsnig et al. (2004); Hubenov (2008a).

\section{MICROPHTHALMA Macquart, 1843}

Microphthalma europaea Egger, 1860 [= Microphthalma disjuncta auct. nec (Wiedemann, 1824)]

Literature: LANGHOFFer (1920); Tschorsnig et al. (2004); Hubenov (2008a).

Minthoini Brauer \& Bergenstamm, 1889

HYPERAEA Robineau-Desvoidy, 1863

Hyperaea sanguinea (Meigen, 1824)

Literature: Strobl (1893); Thalhammer (1899); LANGhoffer (1920); Baranov (1928); Herting (1984); Tschorsnig et al. (2004); Hubenov (2008a).

\section{MINTHO Robineau-Desvoidy, 1830}

Mintho compressa (Fabricius, 1787) [= Wiedemania compressa Fabricius, 1787]

Literature: Frauenfeld (1856); Baranov (1928); Tschorsnig et al. (2004); Hubenov (2008a).

Mintho rufiventris (Fallén, 1817) [= Mintho lacera Rondani, 1847; = Mintho praeceps auct. nec (Scopoli, 1763)]

Literature: Thalhammer (1899); Strobl (1902; 1904); LANGHofFer (1920); Baranov (1928); Tschorsnig et al. (2004); Hubenov (2008a). - Material: Gornji Muć, 29.VI.24.VIII.2014, 1§, 1 ㅇ․

\section{PLESINA Meigen, 1838}

Plesina claripennis Mesnil, 1953

Literature: Lutovinovas et al. (2018).

\section{ZIMINIA Mesnil, 1963}

Ziminia masiceraeformis (Portshinsky, 1881)

Literature: Lutovinovas et al. (2018). 
Neaerini Mesnil, 1966

NEAERA Robineau-Desvoidy, 1830

Neaera atra Robineau-Desvoidy, 1850

Literature: Herting (1984); Tschorsnig et al. (2004); Hubenov (2008a).

Nemoraeini Robineau-Desvoidy, 1863

NEMORAEA Robineau-Desvoidy, 1830

Nemoraea pellucida (Meigen, 1824)

Literature: Strobl (1902; 1904); Langhoffer (1920); Baranov (1928); Ford et al. (2000); Tschorsnig et al. (2004); Hubenov (2008a); Tschorsnig (2017).

Pelatachinini Mesnil, 1966

PELATACHINA Meade, 1894

Pelatachina tibialis (Fallén, 1810)

Literature: Thalhammer (1899); Tschorsnig et al. (2004); Hubenov (2008a).

Polideini Brauer \& Bergenstamm, 1889

LYPHA Robineau-Desvoidy, 1830

Lypha dubia (Fallén, 1810)

Literature: LANGHoffer (1920); BArAnov (1928); Tschorsnig et al. (2004); Hubenov (2008a). - Material: Gornji Muć, 16.III.-10.V.2014, 3ठึ̋̂, 2 우.

Siphonini Rondani, 1844

ACTIA Robineau-Desvoidy, 1830

Actia crassicornis (Meigen, 1824)

Literature: Tschorsnig et al. (2004); Hubenov (2008a). - Material: Gornji Muć, 22.

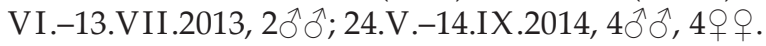

Actia infantula (Zetterstedt, 1844)

Literature: Lutovinovas et al. (2018).

CEROMYA Robineau-Desvoidy, 1830

Ceromya bicolor (Meigen, 1824)

Literature: Sтвовц (1893; 1898; 1904). - Material: Gornji Muć, 27.V.-22.VI.2013, 10ึ, 1; 11.IV.-10.VIII.2014, 3ڤึ̂, 2 ㅇ․

Ceromya flaviseta (Villeneuve, 1921)

Literature: Lutovinovas et al. (2018).

PERIBAEA Robineau-Desvoidy, 1863

Peribaea apicalis Robineau-Desvoidy, 1863

Literature: Tschorsnig et al. (2004); Hubenov (2008a). - Material: Gornji Muć, 29. VI.-20.VII.2014, 1ð.

Peribaea discicornis (Pandellé, 1894)

Literature: Lutovinovas et al. (2018). 
Peribaea longirostris Andersen, 1996

Literature: Lutovinovas et al. (2018).

Peribaea tibialis (Robineau-Desvoidy, 1851)

Literature: Strobl (1893; 1902; 1904); Tschorsnig et al. (2004); Hubenov (2008a). -

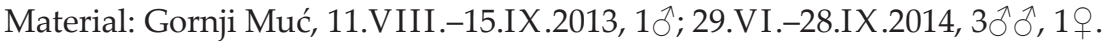

SIPHONA Meigen, 1803

Siphona flavifrons Staeger, 1849

Literature: Tschorsnig et al. (2004); Hubenov (2008a). - Material: Gornji Muć, 22.

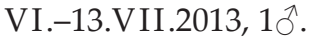

Siphona geniculata (De Geer, 1776)

Literature: LANGHoffer (1920); BARANov (1928); Tschorsnig et al. (2004); Hubenov (2008a).

Siphona hokkaidensis Mesnil, 1957

Literature: Tschorsnig et al. (2004); Hubenov (2008a).

Siphona pauciseta Rondani, 1865

Literature: Lutovinovas et al. (2018).

Siphona rossica Mesnil, 1961

Literature: Herting (1984); Tschorsnig et al. (2004); Hubenov (2008a).

Siphona setosa Mesnil, 1960

Literature: Tschorsnig et al. (2004); Hubenov (2008a).

Siphona variata Andersen, 1982

Literature: Tschorsnig et al. (2004); Hubenov (2008a). - Material: Gornji Muć, 27.V.06.X.2013, 3 우.

Tachinini Robineau-Desvoidy, 1830

\section{CNEPHAOTACHINA Brauer \& Bergenstamm, 1894}

Cnephaotachina danilevskyi (Portshinsky, 1882) [= Cnephaotachina crepusculi Brauer \& Bergenstamm, 1894]

Literature: Coe (1962); Herting (1984); Tschorsnig et al. (2004); Hubenov (2008a).

\section{NOWICKIA Wachtl, 1894}

Nowickia atripalpis (Robineau-Desvoidy, 1863)

Literature: Baranov (1929a); Herting (1984); Tschorsnig et al. (2004); Hubenov (2008a).

Nowickia ferox (Panzer, 1809) [= Fabriciella ferox (Panzer, 1809); = Fabriciella pandellei Baranov 1929]

Literature: THALHAMMER (1899); LANGHOFFER (1920); BARANOv (1928; 1929a); TsCHORSNig et al. (2004); Hubenov (2008a).

Nowickia marklini (Zetterstedt, 1838)

Literature: BARAnov (1929a); Tschorsnig et al. (2004); Hubenov (2008a).

PELETERIA Robineau-Desvoidy, 1830

Peleteria abdominalis Robineau-Desvoidy, 1830

Literature: Tschorsnig et al. (2004); Hubenov (2008a). 
Peleteria ferina (Zetterstedt, 1844)

Literature: Strobl (1893; 1902; 1904); LANGHOFFer (1920); BARANov (1928; 1929a); Tschorsnig et al. (2004); Hubenov (2008a).

Peleteria rubescens (Robineau-Desvoidy, 1830) [= Peleteria nigricornis (Meigen, 1838); = Peleteria tessellata auct. nec (Fabricius)]

Literature: Strobl (1893); Baranov (1928; 1929a); Tschorsnig et al. (2004); Hubenov (2008a).

Peleteria ruficornis (Macquart, 1835)

Literature: Strobl (1893; 1900; 1902; 1904); Thalhammer (1899); LANGHoffer (1920); Baranov (1928); Tschorsnig et al. (2004); Hubenov (2008a).

Peleteria varia (Fabricius, 1794) [= Cyphocera pyrrogaster Rondani, 1859]

Literature: Schiner (1892); Strobl (1893; 1898; 1900); Thalhammer (1899); Baranov (1929a); Rucner (1994); Tschorsnig et al. (2004); Hubenov (2008a).

\section{SCHINERIA Rondani, 1857}

Schineria tergestina Rondani, 1859

Literature: Thalhammer (1899); Tschorsnig et al. (2004); Hubenov (2008a).

\section{TACHINA Meigen, 1803}

Tachina casta (Rondani, 1859) [= Eudoromyia lefebvrei (Robineau-Desvoidy, 1830)]

Literature: LANGHOFFER (1920); BARANOv (1928; 1929a); Tschorsnig et al. (2004); Hu-

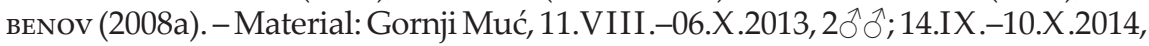
$7 \hat{\jmath}, 3$ 웅.

Tachina fera (Linnaeus, 1761)

Literature: Frauenfeld (1856); Strobl (1898; 1900; 1902; 1904); LANGHoffer (1920); Baranov (1928; 1929a); Coe (1962); Rucner (1994); Britvec (2004); Tschorsnig

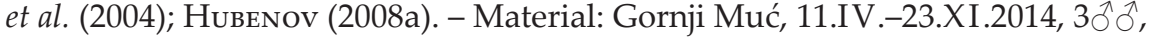
$2 ㅇ$.

Tachina grossa (Linnaeus, 1758)

Literature: Frauenfeld (1860); LANGHOFFER (1920); BARANOv (1928; 1929a); TsCHORSNIG et al. (2004); HuBENOv (2008a).

Tachina lurida (Fabricius, 1781)

Literature: LANGHOFFER (1920); BARANOv (1928; 1929a); TsCHORSNig et al. (2004); HuBENOV (2008a).

Tachina magnicornis (Zetterstedt, 1844) [= Echinomyia tessellata (Fabricius, 1794); = Tachina vernalis auct. nec Robineau-Desvoidy, 1863]

Literature: Frauenfeld (1856); Schiner (1862); Strobl (1902; 1904); LANGHoffer (1920); Baranov (1928; 1929a; 1930a); Coe (1962); Rucner (1994); Tschorsnig et al. (2004); Hubenov (2008a); Tschorsnig (2017). - Material: Gornji Muć, 11.IV.24.V.2014, 2ðえ.

Tachina praeceps Meigen, 1824

Literature: Frauenfeld (1860); Strobl (1893; 1898; 1900); Baranov (1929a); Herting (1960); Tschorsnig et al. (2004); Hubenov (2008a); TsCHORSNig (2017).

Tachina ursina Meigen, 1824

Literature: LANGHOFFer (1920); BARANOv (1928; 1929a); Tschorsnig et al. (2004); HuBENOV (2008a). 


\section{DISCUSSION}

A previous checklist of Croatian Tachinidae included 241 species (HubEnov, 2008a); this checklist, however, lacks annotations, and also ignores some sources that contain Croatian records. We considered 307 species of Tachinidae in the present study, involving over 100 references, which validated about presences of the species discussed; recently collected material accounted for almost the third of the number of species (100 species), with precise localities, dates and numbers of specimens added, which also supported some of the older references, when these were not supplemented by recent ones. Thus, the following 7 species based solely on older Croatian records have been confirmed by the present study: Ceromya bicolor, Epicampocera succincta, Gastrolepta anthracina, Medina melania, Ocytata pallipes, Periscepsia carbonaria, and Wagneria cunctans. However, records from those older sources, which have not been confirmed by more recent and reliable studies, were considered unreliable, and in that way 32 taxa were excluded from the main species list, 29 as doubtful records, and 3 as doubtful taxa, all reviewed in Appendix 1. The occurrences of the following 10 doubtfully recorded and excluded species from the Croatian fauna tend to indicate their widespread distribution in the Balkans, which makes their presence in Croatia very probable: Bithia modesta, Erycia festinans, Exorista mimula, Hubneria affinis, Lydina aenea, Macquartia grisea, Medina luctuosa, Siphona cristata, Winthemia erythrura and W. variegata; on the other hand, the following 2 doubtfully recorded species in Croatia are unverified from the Balkans by more recent and reliable studies, which would tend to indicate that their presence in Croatia is very unlikely: Hyperaea femoralis and Nilea rufiscutellaris (see Appendix 1).

In this report, Croatia is presented as the fourth country in the region, after Bulgaria, Greece and Romania, with respect to faunistic data (Tab. 2). However, these data do not reflect actual biodiversity, but rather the level of investigation, since Bulgaria is the site of the most intensive research, whereas other countries in the region remain insufficiently studied and require further investigation. Most known species of Croatian tachinid fauna are more eurybiotic and widely distributed in the Palaearctic Region (Hubenov, 2008a). However, a considerable number of species exhibit a Mediterranean type of distribution, which is due to the relatively large part of the littoral zone owned by this country in comparison to the area that is situated deeper inland. In general, Croatia has a considerably higher than average representation of Mediterranean and South Palaearctic-Palaeotropical species than other Balkan countries and ranks second after Greece (see Hubenov, 2008a).

Croatia hosts thirteen tachinid species (mostly of the Southern type of distribution) unknown in other parts of the Balkans: Bithia proletaria, Campylocheta confusa, Chetogena micronychia, Exorista florentina, E. unicolor, Hyperaea sanguinea, Istocheta subcinerea, I. sublutescens, Meigenia simplex, Leucostoma edentatum, Peribaea discicornis, P. longirostris and Ramonda plorans; among these enumerated taxa, one species (Campylocheta confusa) is endemic to Croatia, another two (Bithia proletaria, Exorista unicolor) are otherwise known only from Italy, and another one (Istocheta sublutescens) is otherwise known only from Switzerland as far as it is known at all (Herting \& Dely-Draskovits, 1993; Tschorsnig et al., 2004; Cerretti, 2010). A further seven Mediterranean and South Palaearctic-Palaeotropical species are known in the Balkans only from Croatia and Greece: Besseria zonaria, Bithia golanensis, Ceratochaetops delphinensis, Euthera fascipennis, Phryxe caudata, Plesina claripennis and Stomina calvescens (Cerretti \& Ziegler, 2004; Hubenov, 2008a; 2008b; PohjoismäKi et al., 2016; Zeegers, 2017; Lutovinovas et al., 2018). Howe- 
ver, Croatia is not a large country and the level of knowledge regarding the fauna of some countries in this region is very poor, and especially of the western peninsular countries bordering on Croatia (Slovenia and Bosnia \& Herzegovina, but also Macedonia and Albania; see Tab. 2); this suggests that some rarely collected species could possibly be recorded in nearby countries in the future. It also seems fair to say that the Croatian tachinid fauna can be expected to be much richer than currently known.

Tab. 2. Tachinidae fauna in Balkan countries*

\begin{tabular}{|c|l|c|c|}
\hline Rank & State & No. of species (2018) & Balkan context (\%) \\
\hline 1 & Bulgaria & 409 & 66.6 \\
\hline 2 & Greece & 340 & 55.4 \\
\hline 3 & Romania & 330 & 53.7 \\
\hline 4 & Croatia & 307 & 50.0 \\
\hline 5 & Serbia \& Montenegro** & 290 & 47.2 \\
\hline 6 & Slovenia & 169 & 27.5 \\
\hline 7 & Bosnia \& Herzegovina & 92 & 15.0 \\
\hline 8 & Macedonia & 87 & 14.2 \\
\hline 9 & Albania & 65 & 10.6 \\
\hline & total & 614 & 100.0 \\
\hline
\end{tabular}

*After Groot et al. (2007), Predovnik \& Tschorsnig (2007), Hubenov (2008a, 2008b), Ziegler (2010), Robertson \& Shaw (2012), Stanković et al. (2014), Ронjoismäki et al. (2016), Zeegers (2017), and the present publication.

**Published sources provide a cumulative number of species, consisting of a former state that comprised Serbia, Montenegro and Kosovo.

\section{ACKNOWLEDGEMENTS}

We are indebted to our Czech colleagues, J. Halada, M. Kadlecová, and M. Kafka, for kindly supplementing our studies with valuable material from their private collections. We also thank H.-P. Tschorsnig (Stuttgart) for additional literature sources and for the confirmation of several identifications. This paper was supported by an $S$ grant of MSMT-CZ (Ministry of Education, Sports and Youth of the Czech Rep. for M. Barták.

Received August 17, 2017

\section{REFERENCES}

Al-DobaI, S., ReItz, S. \& Sivinski, J., 2012: Tachinidae (Diptera) associated with flowering plants: estimating floral attractiveness. Biological Control 61, 230-239.

Androić, M., 1956: Contribution à l'étude de Cnethocampa pityocampa Schiff. Revue de Pathologie végétale et d'Entomologie agricole de France 35, 251-262.

Androić, M., 1957: La processionaire du pin (Cnethocampa pityocampa Schiff.). Étude biologique et écologique. Glasnik za Sumske pokuse 13, 351-459. [In Croatian with French summary.]

Baranov, N., 1928: Tachiniden-sammlung des Zoologischen Museums in Zagreb. Glasnik Hrvatskog Prirodoslovnog Društva 39/40, 196-200.

BARANOv, N., 1929a: Studien an pathogenen und parasitischen Insekten I. Die jugoslavischen Arten der Tachinidengruppe Echinomyia. (Fauna der jugoslavischen Tachiniden I). Arbeiten aus der Parasitologischen Abteilung Institut für Hygiene und Schule für Volksgesundheit in Zagreb 1, 1-23. 
BARANOv, N., 1929b: Studien an pathogenen und parasitischen Insekten II. Betrag zur Kenntnis der Phasiinen mit besonderer Berücksichtigung der Gruppe Ocyptera (Diptera, Tachin.). Arbeiten aus der Parasitologischen Abteilung Institut für Hygiene und Schule für Volksgesundheit in Zagreb 2, 1-22.

Baranov, N., 1929c: A contribution to the morphology of the Tachinid flies bred from Pyrausta nubilalis $\mathrm{Hb}$. Scientific Reports - international Corn Borer Investigations 2, 128-130.

Baranov, N., 1930a: Die Sternitenkette des Abdomens bei den parasitären Raupenfliegen und ihre systematische Bedeutung. Zeitschrift für Parasitenkunde 2/4, 506-534.

Baranov, N., 1930b: O Eutachina civilis Rnd., parazitu metlice. Glasnik Jugoslovenskog entomološkog društva 5/6, 62-65.

BARANov, N., 1930c: O pravom specifičnom nazivu tachine roda Ceromasia (Rndn.) Lundbeek, koja parazitira na Pyrausta nubilalis. Glasnik Ministarstva poljoprivrede (Beograd) 29, 111-112.

BARANOv, N., 1931: Studien an pathogenen und parasitischen Insekten III. Betrag zur Kenntnis der Raupenfliegengattung Carcelia R.-D. Arbeiten aus der Parasitologischen Abteilung Institut für Hygiene und Schule für Volksgesundheit in Zagreb 3, 1-45.

BARANOv, N., 1932: Über die aus Wiesenzünslerraupen gezogene Larvaevoride Eutachina civilis Rnd. Acta Societatis entomologicae jugoslavicae 5/6, 62-65.

Baranov, N. \& Hergula, B., 1928: Über die systematische Stellung der aus Pyrausta nubilalis Hb. erzogenen Tachine Ceromasia senilis (Mg.) auct. nov. Glasnik hrvatskoga prirodoslovnoga Društva 39/40, 192-195.

BARTÁK, M. \& KoKAn, B., 2017: The first record of Rhamphomyia (Pararhamphomyia) intersita (Diptera: Empididae) in Europe. Natura Croatica 26(2), 325-330.

Bergström, C. \& Bartsch, H., 2005: Phasia barbifrons (Girschner, 1887) - en småväxt parasitfluga (Dipt., Tachinidae) ny för Norden. Entomologisk Tidskrift 126, 187-190.

BJegović, P., 1957: Žitni bauljar (Zabrus tenebrionides Goeze) i njegova parazitiska muva (Viviania cinerea Fall.). Institut za Zaštitu Bilja, Beograd Posebna Izdanja 5, 104 pp.

Brauer, F., 1898: Beiträge zur Kenntniss der Muscaria schizometopa. Sitzungsberichte. Kaiserliche Akademie der Wissenschaften in Wien 107, 493-546.

Brauer, F. \& Bergenstamm, J.E.V., 1889: Die Zweiflüger der Kaiserliche Museums zu Wien, 4. Denkschriften der Kaiserlichen Akademie der Wissenschaften in Wien 56/1, 69-180.

Brauer, F. \& Bergenstamm, J.E.V., 1891: Die Zweiflüger der Kaiserliche Museums zu Wien, 5. Denkschriften der Kaiserlichen Akademie der Wissenschaften in Wien 58, 305-446.

Brauer, F. \& Bergenstamm, J.E.V., 1894: Die Zweiflügler des Kaiserlichen Museums zu Wien. VII. Vorarbeiten zu einer Monographie der Muscaria Schizometopa (exclusive Anthomyidae), Pars. IV. Denkschriften der Kaiserlichen Akademie der Wissenschaften in Wien, mathematisch-naturwissenschaftliche Classe 61, 537-624.

BRIschKe, C.G.A., 1885: Meine erzogenen parasitisch lebenden Fliegen. Schriften der naturforschenden Gesellschaft Danzig (2) 6, 15-22.

BRITVEC, B., 2000: Dvokrilci (Diptera) značajni za poljoprivredu i šumarstvo Hrvatske u svjetlu faunističkih istraživanja. Agronomski Glasnik 5-6, 323-343.

BRITVEC, B., 2004: Dvokrilci (Diptera) značajny za poljoprivredu i šumarstvo Hrvatske u svjetlu faunističkih istraživanja - dodatak. Agronomski Glasnik 6, 413-427.

Burgess, A.F. \& Crossman, S.S., 1929: Imported insect enemies of the gipsy moth and the brown-tail moth. Technical Bulletin of the United States Department of Agriculture 86, $147 \mathrm{pp}$.

BystrowsKI, C., 2012: Thoughts on how Trichopoda pennipes (F.) reached Israel. In O'HarA J.E. (ed.), The Tachinid Times 25, 13-15.

Cerretti, P., 2005: Revision of the West Palaearctic species of the genus Pales Robineau-Desvoidy (Diptera: Tachinidae), Zootaxa 885, 1-36.

Cerretti, P., 2010: I tachinidi della fauna italiana (Diptera Tachinidae), con chiave interattiva dei generi ovest-paleartici (2 volumes), $573+339$ pp. Centro Nazionale Biodiversitá Forestale, Cierre Edizioni, Verona.

Cerretti, P. \& Ziegler, J., 2004: Chorologic data on tachinid flies from mainland Greece (Diptera, Tachinidae). Fragmenta Entomologica 362, 275-317.

Cerretti, P. \& Tschorsnig, H.-P., 2008: A new species of Plesina Meigen (Diptera: Tachinidae) from the Mediterranean. Stuttgarter Beiträge zur Naturkunde A, Neue Serie 1, 445-450.

Cerretti, P. \& Shima, H., 2011: World revision of Dolichocolon Brauer \& Bergenstamm (Diptera: Tachinidae: Exoristinae: Goniini). Zoological Journal of the Linnean Society 162(3), 544-584. 
Coe, R.L., 1960: Diptera taken in Jugoslavia from May to July, 1955, with localities and notes. Part three. Glasnik Prirodnjačkog muzeja u Beogradu (B) 15, 153-173.

Coe, R.L., 1962: A further collection of Diptera from Jugoslavia, with localities and notes. Part two. Glasnik Prirodnjačkog muzeja u Beogradu (B) 18, 95-136.

Cooper, B.E. \& O'Hara, J.E., 1996: Diptera types in the Canadian National Collection of Insects. Part 4 Tachinidae, Biological Resources Division, 1918/B, 94 pp.

Dowden, P.B., 1933. Lydella nigripes and L. piniariae, fly parasites of certain tree-defoliating caterpillars. Journal of Agricultural Research 46, 963-995.

Ford, T.H., Shaw, M.R., Robertson, D.M., 2000: Further host records of some West Palaearctic Tachinidae (Diptera). Entomologist's Record and Journal of Variation 112, 25-36.

Francati, S., Dindo, M.L. \& Cerretti, P., 2017: A new host record for Euthera fascipennis (Diptera: Tachinidae). Fragmenta entomologica 49(1), 93-95.

Frauenfeld, G., 1856: Beitrag zur Fauna Dalmatiens. Verhandlungen des Zoologisch-botanischen Vereins in Wien 6, 431-448.

Frauenfeld, G., 1860: Weiterer Beitrag zur Fauna Dalmatiens. Verhandlungen des Zoologisch-botanischen Vereins in Wien 10, 787-794.

Gilasian, E., Talebi, A.A., Ziegler, J., Manzari, S. \& Parchami-Araghi, M., 2013: A review of the genus Cylindromyia Meigen (Diptera: Tachinidae) in Iran, with the description of two new species and the newly discovered male of C. persica Tschorsnig. Studia dipterologica 20(2), 299-324.

Groot, M. De, Virant-Doberlet, M. \& Žunić, A., 2007: Trichopoda pennipes F. (Diptera, Tachinidae): a new natural enemy of Nezara viridula (L.) in Slovenia - short communication. Agricultura (Slovenia) 5, 25-26.

Hadžistević, D., 1961: A study of natural enemies of the Durra stem borer (Sesamia cretica Led.). Zaštita Bilja 12: 105-112. [In Croatian with English summary.]

Hergula, B., 1928: Observations on the Corn Borer in Jugoslavia. Scientific Reports - international Corn Borer Investigations 1, 201-222.

Hergula, B., 1929: Insect Parasites of the Corn Borer in Northern Jugoslavia. Scientific Reports - International Corn Borer Investigations 2, 111-127.

Herting, B., 1960: Biologie der westpaläarktischen Raupenfliegen (Dipt., Tachinidae). Monographien zur angewandten Entomologie 16, 188 pp., Berlin (Parey).

Herting, B., 1974: Revision der von J. Egger, J.R. Schiner, F. Brauer und J.E. Bergenstamm beschriebenen europäischen Tachiniden und Rhinophorinen (Diptera). Naturkundliches Jahrbuch Der Stadt Linz 1974, 129-145.

Herting, B., 1975: Nachträge und Korrekturen zu den von Meigen und Rondani beschriebenen Raupenfliesgen (Dipt. Tachinidae). Stuttgarter Beiträge zur Naturkunde, Serie A (Biologie) 271, 1-13.

Herting, B., 1983: Phasiinae. In Lindner, E. (ed.), Die Fliegen der palaearktischen Region 9, 1-88.

Herting, B., 1984: Catalogue of Palearctic Tachinidae (Diptera). Stuttgarter Beiträge zur Naturkunde, Serie A (Biologie) 369, 1-228.

Herting, B., 1985: Tachinidae. In Haeselbarth, E. (ed.), Determination list of entomophagous insects Nr. 10. International Organization for Biological and Integrated Control of Noxious Animals and Plants/ West Palaearctic Regional Section Bulletin 8(4), 61 pp.

Herting, B., 1993: Tachinidae. In Vidal, S. (ed.), Determination list of entomophagous insects Nr. 12. International Organization for Biological and Integrated Control of Noxious Animals and Plants/West Palaearctic Regional Section Bulletin 16(3), 56 pp.

Herting, B., 2017: A critical revision of host records of Palearctic Tachinidae (Diptera) until 1937. Stuttgarter Beiträge zur Naturkunde, Serie A (Biologie): 41-173.

Herting, B. \& Dely-Draskovits, A., 1993: Family Tachinidae. In Soós, A. \& Papp, L. (eds.), Catalogue of Palaearctic Diptera 13. Anthomyiidae-Tachinidae, pp. 118-624. Budapest (Academy Press).

Hubenov, Z., 2008a: Composition and Zoogeographical characteristics of the family Tachinidae (Insecta: Diptera) in the Balkan countries. Acta Zoologica Bulgarica 60(3), 243-265.

Hubenov, Z., 2008b: Composition and zoogeographical characteristics of the family Tachinidae (Diptera: Insecta) in Serbia and Bulgaria. In Makarov, S.E. \& Dimitrijević, R.N. (eds.), Advances in Arachnology and Developmental Biology 12, 375-394.

Hubenov, Z., 2015: Comparative Zoogeographical Review of the Tachinid Fauna (Diptera: Tachinidae) of the Belasitsa and Slavyanka Mountains. Acta Zoologica Bulgarica 67(3), 351-364. 
Inclán, D.J., Cerretti, P. \& Marini, L., 2014: Interactive effects of area and connectivity on the diversity of tachinid parasitoids in highly fragmented landscapes. Landscape Ecology 29, 879-889.

Inclán, D.J., Cerretti, P. \& Marini, L., 2015: Landscape composition affects parasitoid spillover. Agriculture, Ecosystems and Environment 208, 48-54.

Inclán, D.J., Dainese, M., Cerretti, P., Paniccia, D. \& Marini, L., 2016: Spillover of tachinids and hoverflies from different field margins. Basic and Applied Ecology 17, 33-42.

Keglević, S., 1970: Inventarizacija štetnika I bolesti bilja na graničnom pojasu uz jugoslavensko-mađarsku granicu na području Hrvatske u 1969. godini, Republički sekretarijat za privredu SR Hrvatske: Granična karantenska služba za zastitu bilja, 65 pp., Zagreb.

KovačEvić, Ž., 1926: Der Ringelspinner und der Schwammspinner und ihre Parasiten. Anzeiger für Schädlingskunde 2, 93-94.

KovačEvić, Ž., 1928: Withering of oak trees in Posavina from the entomological and biological viewpoint. Šumarski List 52/4, 182-195, 52/5, 225-242. [In Croatian with French abstract.]

KrivosheinA, M. \& Richter, V.A., 2015: Tachinid flies (Diptera: Tachinidae), pollinators of aboriginal and alien species of the umbel plants (Apiaceae) in Moscow Region (Russia). Caucasian Entomological Bulletin 11, 215-220. [In Russian with English abstract.]

Kuhlmann, L., 1890: Noch einmal Acherontia atropos. Entomologische Zeitschrift, Frankfurt a. Main 4, 73-74.

LANGHoffer, A., 1896: Entomološki pabirci s puta Margite uz neke druge entomološke podatke za Dalmaciju. Glasnik Hrvatskog Naravoslovnog Društva 9, 346-358.

Langhoffer, A., 1920: Beitrage zur Dipterenfauna Kroatiens (Fortsetzung). Glasnik Hrvatskoga Prirrodoslovnoga Društva 32, 57-63.

Loww, H., 1854: Neue Beiträge zur Kenntnis der Dipteren. Zweiter Beitrag. Programm der Königlichen Realschule zu Meseritz 1854, 1-24.

Lutovinovas, E., Barták, M., Kokan, B. \& Ozimec, R., 2018: An update to the Tachinidae fauna of Croatia (Diptera). Entomologica Fennica 29, 54-60.

MacelJski, M. \& BAlarin, I., 1975: Paraziti sovice game (Autographa gamma L.) u svijetu i Jugoslaviji. Acta entomologica Jugoslavica 11/1-2, 109-124.

Melis, A., 1940: Contributo alla conoscenza del Bombice del Pino (Dendrolimus pini L.). Redia 26, 73-175.

Mesnil, L.P., 1944-1975: Larvaevorinae (Tachininae). In Lindner, E. (ed.), Die Fliegen der palaearktischen Region 10(1-3), 1-1435.

MıнÁLyI, F., 1986: Fürkészlegyek-Aszkalegyek Tachinidae - Rhinophoridae. Fauna Hungarica 161, 425 pp. Budapest (Akadémiai Kiadó).

Mückstein, P., Tschorsnig, H.-P., VañHara, J. \& Michalková, V., 2007: New host and country records for European Tachinidae (Diptera). Entomologica Fennica 18, 179-183.

NovaK, A., 1899: No title. Entomologische Zeitschrift, Frankfurt a. Main 13, 90.

O'Hara, J.E. \& Wood, D.M., 2004: Catalogue of the Tachinidae (Diptera) of America north of Mexico. Memoirs on Entomology, International 18, iv + 410 pp., Ottava.

O'Hara, J.E., Shima, H. \& Zhang, C.-T., 2009: Annotated Catalogue of the Tachinidae (Insecta: Diptera) of China. Zootaxa 2190, 1-236.

O'Hara, J.E. \& Cerretti, P., 2016: Annotated catalogue of the Tachinidae (Insecta, Diptera) of the Afrotropical Region, with the description of seven new genera. Zookeys 575, 1-344.

PAbst, M., 1890a: Ein unheimlicher Gast auf Deutschlands Fluren. Entomologische Zeitschrift, Frankfurt a. Main 3, 131-132, 137-138.

PAвsт, M., 1890b: Zur “vorläufigen Stellungnahme” des Herrn Dr. Fuchs. Entomologische Zeitschrift, Frankfurt a. Main 4, 64-65.

Pape, T., Blagoderov, V. \& Mostovski, M.B., 2011: Order Diptera Linnaeus, 1758. In Zhang Z.-Q. (ed.), Animal biodiversity: An outline of higher-level classification and survey of taxonomic richness. Zootaxa 3148, 222-229.

Parker, H.L., VAnce, A.M., Smith, H.D. \& Gamkrelidze, W., 1929: Pyrausta nubilalis Hubn. in Europe: notes on infestation and parasitism from 1926 to 1928, Journal of economic Entomology 22, 688-693.

Pernek, M., Lacković, N. \& Matošević, D., 2013: Biology and natural enemies of spotted ash looper, Abraxas pantaria (Lepidoptera, Geometridae) in Krka National Park. Periodicum biologorum 115, 371-377. 
Pernek, M., Lukić, I., Lacković, N., Cota, E. \& Tschorsnig, H.-P., 2016: Tachinid (Diptera: Tachinidae) parasitoids of spotted ash looper (Abraxas pantaria) in Krka National Park in Croatia. Periodicum Biologorum 117(4), 533-535.

Pétremand, G., Rochefort, S., Jaccard, G. \& Fischer, S., 2015: First detection of the southern green stink bug parasitoid Trichopoda pennipes (Fabr.) (Diptera: Tachinidae) in Western Switzerland. Mitteilungen der schweizerischen entomologischen Gesellschaft 88, 403-409.

Ронjoismäki, J.L.O, Kahanpä̈̈, J. \& Mutanen, M., 2016: DNA Barcodes for the Northern European Tachinid Flies (Diptera: Tachinidae). PLoS ONE 11(11), e0164933. doi:10.1371/journal. pone.0164933

Predovnik, Ž. \& Tschorsnig, H.-P., 2007: Tachinidae (Diptera) reared from clearwing moths (Lepidoptera: Sesiidae) in Slovenia. Acta entomologica slovenica 15, 47-50.

Radović, J., Čivić, K., Topić, R. \& Vukelić, V.P. (eds.), 2009: Biodiversity of Croatia. Second revised edition. State Institute for Nature Protection Ministry of Culture, Republic of Croatia, Zagreb, $43 \mathrm{pp}$.

Riedel, M.P., 1908: Beitrag zur Biologie der Musciden. Entomologisches Wochenblatt 25, 133-134.

Rieger, C. \& Tschorsnig, H.-P., 2001: Neue Wirtsbefunde von Raupenfliegen (Diptera: Tachinidae) aus Wanzen (Heteroptera: Pentatomidae und Rhopalidae). Mitteilungen des entomologischen Vereins Stuttgart 36, 22.

Robertson, D.M. \& Shaw, M.R., 2012: Further rearing records of some West Palaearctic Tachinidae (Diptera). Entomologist's Gazette 63, 161-172.

Rubin, G., 2000: Parasite complex of Thaumetopoea pityocampa Schiff. in the forest district Split. Šumarski List 124, 363-372. [In Croatian with German summary.]

RuCNER, Z., 1994: Beitrag zur Entomofauna einiger Waldassoziationen Kroatiens. Natura Croatica 3(1), $1-22$.

Salerno G., Colazza, S. \& Bin, F., 2002: Nezara viridula parasitism by the tachinid fly Trichopoda pennipes ten years after its accidental introduction into Italy from the New World. BioControl 47, 617-624.

Schiner, J.R., 1862: Fauna Austriaca. Die Fliegen (Diptera). Druck und Verlag von Carl Gerold's Sohn. 674 pp., Wien.

Siebold, C.T.E. von, 1843: IX. Bemerkungen über eine den Bacillus Rossii bewohnende Schmarotzer-Larve. Zeitschrift für Entomologie 4, 389-394.

Stanković, S.S., Žıкić, V., Hrić, B. \& Tschorsnig, H.-P., 2014: Several records of Tachinidae (Diptera) reared from their hosts in Serbia and Montenegro. Biologica Nyssana 5, 71-73.

STEIN, P., 1924: Die verbreitetsten Tachiniden Mitteleuropas nach ihren Gattungen und Arten. Archiv für Naturgeschichte 90(A), 1-271.

Stireman, J.O., O'Hara, J.E. \& Wood, D.M., 2006: Tachinidae: evolution, behavior, and ecology. Annual Review of Entomology 51, 525-555.

Strobl, G., 1893: Beiträge zur Dipterenfauna des österreichischen Littorale. Wiener Entomologische Zeitung 12(3), 89-108.

Strobl, G., 1898: Fauna diptera Bosne, Hercegovine i Dalmacije. Glasnik Zemaljskog muzejau Bosni i Hercegovini 10, 387-616.

Strobl, G., 1900: Dipterenfauna von Bosnien, Herzegovina und Dalmatien. Wissenschaftliche Mittheilungen aus Bosnien und der Hercegovina 7, 552-670.

StroвL, G., 1902: Novi prilozi fauni diptera Balkanskog poluostrva. Glasnik Zemaljskog muzeja u Bosni i Hercegovini 14(3/4), 461-518.

SтRовL, G., 1904: Neue Beiträge zur Dipterenfauna der Balkanhalbinsel. Wissenschaftliche Mittheilungen aus Bosnien und der Hercegovina 9, 519-581.

Sun, X.-K. \& Marshall, S.A., 2003: Systematics of Phasia Latreille (Diptera: Tachinidae). Zootaxa 276, $1-320$.

Thalhammer, J., 1899: Ordo Diptera. In: Fauna Regni Hungariae: Animalium Hungariae huqusque cognitorum enumeratio systematica. III. Arthropoda, 78 pp.

TOOKER, J.F., HAuser, M. \& HANKs, L.M. 2006. Floral host plants of Syrphidae and Tachinidae (Diptera) of Central Illinois. Annals of the Entomological Society of America 99(1), 96-112.

TöLG, F. \& FAHRINGER, J., 1911: Beitrag zur Dipteren und Hymenopteren fauna Bosniens, der Herzegowina und Dalmatiens. Mitteilungen des naturwissenschaftlichen Vereins an der Universitat Wien 9(1), $1-14$.

Tschorsnig, H.-P., 1990: Raupenfliegen aus dem Museum Wiesbaden (Diptera, Tachinidae). Mitteilungen des internationalen entomologischen Vereins Frankfurt/Main 15, 91-122. 
Tschorsnig, H.-P., 2005: Tachinidae. In BAur H. (ed.), Determination list of entomophagous insects Nr. 14. International Organization for Biological and Integrated Control of Noxious Animals and Plants/ West Palaearctic Regional Section Bulletin 28(11), 3-20.

Tschorsnig, H.-P., 2017: Preliminary host catalogue of Palaearctic Tachinidae (Diptera), http://www. nadsdiptera.org/Tach/WorldTachs/CatPalHosts/Cat_Pal_tach_hosts_Ver1.pdf

Tschorsnig, H.-P. \& Herting, B., 1994: Die Raupenfliegen (Diptera: Tachinidae) Mitteleuropas: Bestimmungstabellen und Angaben zur Verbreitung und Ökologie der einzelnen Arten. Stuttgarter Beiträge zur Naturkunde, Serie A (Biologie) 506, 1-170.

Tschorsnig, H.-P. \& Herting, B., 2005: Die Raupenfliegen-Sammlung Friedrich A. Wachtl (Diptera: Tachinidae). Veröffentlichungen des Tiroler Landesmuseums Ferdinandeum 84, 181-236.

Tschorsnig, H.-P., Richter, V.A., Cerretti, P., Zeegers, T., Bergström, C., Vañhara, J., Van De Weyer, G., Bystrowski, C., Raper, C., Ziegler, J. \& Hubenov, Z., 2004: Tachinidae. In Pape, T. (ed.), Diptera: Brachycera, Fauna Europaea Service, http://www.faunaeur.org

WAснтL, F.A., 1889: Biologische und synonymische Notizen über parasitisch lebende Dipteren. Wiener entomologische Zeitung 8, 57-59.

Zeegers, T., 2017: New and interesting Tachinidae from Greece. In O'Hara J.E. (ed.), The Tachinid Times $30,21-25$.

ZIEGLER, J., 1996: Campylocheta fuscinervis auctorum - ein Artenkomplex (Diptera, Tachinidae). Studia Dipterologica 3, 311-322.

ZIEGLER, J., 2010: Revision of the genus Germaria Robineau-Desvoidy (Diptera, Tachinidae) from Greece, with descriptions of two new species. Deutsche Entomologische Zeitschrift 57(1), 43-57.

ZIEGLER, J., 2011: Rezente Arealerweiterungen bei Wanzenfliegen (Diptera: Tachinidae, Phasiinae) in Nordostdeutschland und eine Übersicht zur Gesamtverbreitung von fünf Arten. Studia dipterologica $18(1 / 2), 29-54$.

Ziegler, J., Lutovinovas, E. \& Zhang, C.-T., 2016: The taxa of the Dinera carinifrons species complex (Diptera, Tachinidae), with the description of a new West Palaearctic subspecies and three lectotype designations. In Ziegler, J. (ed.), Diptera Stelviana 2. Studia Dipterologica. Supplement 21, $249-275$.

\title{
SAŽETAK
}

\section{Ažurirani popis hrvatskih muha gusjeničarki (Diptera: Tachinidae)}

\author{
E. Lutovinovas, R. Ozimec, M. Barták \& B. Kokan
}

U radu smo obradili 307 vrsta muha gusjeničarki (Tachinidae), uključujući literaturne navode koji potvrđuju prisutnost navedenih vrsta, pri čemu smo uključili i novosakupljeni materijal koji sadrži trećinu poznatih vrsta (100 vrsta), s preciznim lokalitetima, datumima i brojem primjeraka, a koji podržava navode nekih starih referenci, koje međutim nisu podržale novije studije. Sljedećih sedam vrsta, poznatih isključivo iz starijih hrvatskih referenci potvrđeno je ovim radom: Periscepsia carbonaria, Wagneria cunctans, Gastrolepta antracina, Medina melania, Epicampocera succincta, Ocytata pallipes i Ceromya bicolor. Međutim, navodi istih starijih referenci koji nisu potvrđeni novijim i pouzdanim studijama ili nalazima, definirani su kao nepouzdani i izbačeni iz konačnog popisa. Tako su isključene 32 svojte, 29 kao sumnjivi navodi, a 3 kao taksonomski sumnjive svojte, što je napomenuto i raspravljeno. U Hrvatskoj obitava trinaest vrsta muha gusjeničarki (uglavnom južne rasprostranjenosti) koje ne nalazimo u drugim dijelovima Balkana: Bithia proletaria, Campylocheta confusa, Chetogena micronychia, Exorista florentina, E. unicolor, Hyperaea sanguinea, Istocheta subcinerea, I. sublutescens, Meige- 
nia simplex, Leucostoma edentatum, Peribaea discicornis, P. longirostris i Ramonda plorans. Međutim, Hrvatska nije velika zemlja, a razina znanja o fauni nekih zemalja ove regije vrlo je loša; što sugerira da neke rijetke vrste u budućnosti mogu biti zabilježene i u obližnjim zemljama. Također možemo očekivati kako će hrvatska fauna muha gusjeničarki u konačnici biti puno bogatija nego što je trenutno poznato. 
Appendix 1. Doubtful records and doubtful taxa of Croatian Tachinidae with their occurrences in nearby countries with references:

\section{DeXIINAe}

Billaea fortis (Rondani, 1862)

Literature: LANGHOFfer (1920). - Nearby countries: Bulgaria (Tschorsnig et al., 2004; Hubenov, 2008a, 2008b, 2015).

Campylocheta praecox (Meigen, 1824) [= Campylochaeta obscura auct. nec (Fallén, 1810)]

Literature: LANGHoffer (1920). - Nearby countries: Greece, Serbia \& Montenegro* (TschorSNig et al., 2004; Hubenov, 2008a, 2008b).

Halidaya aurea Egger, 1856

Literature: Frauenfeld (1860). - Nearby countries: Bulgaria, Romania (Tschorsnig et al., 2004; Hubenov, 2008a, 2008b, 2015).

Thelaira leucozona (Panzer, 1809)

Literature: Thalhammer (1899). - Nearby countries: Bulgaria, Serbia \& Montenegro* (TsCHORSNig et al., 2004; Hubenov, 2008a, 2008b).

\section{EXORISTINAE}

Admontia podomyia Brauer \& Bergenstamm, 1889 [= Admontia amica auct. nec (Meigen)]

Literature: LANGHOFFER (1920); BARANov (1928). - Nearby countries: Bulgaria (Tschorsnig et al., 2004; Hubenov, 2008a, 2008b, 2015).

Admontia seria (Meigen, 1824) [= Trichoparia seria (Meigen, 1824)]

Literature: Keglević (1970). - Nearby countries: Romania (Tschorsnig et al., 2004; Hubenov, 2008a).

Carcelia tibialis (Robineau-Desvoidy, 1863)

Literature: Rucner (1994). - Nearby countries: Bulgaria (Tschorsnig et al., 2004; Hubenov, 2008a, 2008b).

Chetogena media Rondani, 1859

Literature: BARANov (1928). - Nearby countries: Greece, Serbia \& Montenegro* (Tschorsnig et al., 2004; Hubenov, 2008a, 2008b).

Drino lota (Meigen, 1824) [= Exorista lota Meigen, 1824]

Literature: STRoвL (1900). - Nearby countries: Bulgaria, Romania, Serbia \& Montenegro* (TsCHORSNig et al., 2004; HubENOv, 2008a, 2008b, 2015).

Erycia festinans (Meigen, 1824) [= Ceromasia festinans Meigen, 1824]

Literature: Strobl (1893; 1900); Baranov (1928). - Nearby countries: Bulgaria, Greece, Romania, Slovenia (Tschorsnig et al., 2004; Hubenov, 2008a, 2008b, 2015).

Exorista mimula (Meigen, 1824) [= Tachina erucarum Rondani, 1859]

Literature: Strobl (1893); Thalhammer (1899); Baranov (1928). - Nearby countries: Bulgaria, Greece, Romania, Serbia \& Montenegro*, Slovenia (Tschorsnig et al., 2004; Hubenov, 2008a, 2008b, 2015).

Gonia atra Meigen, 1826

Literature: Frauenfeld (1856); Schiner (1862); Strobl (1898; 1900). - Nearby countries: Bulgaria (Tschorsnig et al., 2004; Hubenov, 2008a, 2008b). 
Hubneria affinis (Fallén, 1810) [= Exorista affinis Fallén, 1810]

Literature: LANGHoffer (1920). - Nearby countries: Bulgaria, Greece, Macedonia, Romania, Serbia \& Montenegro*, Slovenia (Tschorsnig et al., 2004; Hubenov, 2008a, 2008b, 2015).

Medina luctuosa (Meigen, 1824)

Literature: LANGHoffer (1920); Baranov (1928). - Nearby countries: Bulgaria, Macedonia, Romania, Serbia \& Montenegro* (Tschorsnig et al., 2004; Hubenov, 2008a, 2008b, 2015).

Meigenia incana (Fallén, 1810)

Literature: Keglević (1970). - Nearby countries: Bulgaria, Greece, Romania (Tschorsnig et al., 2004; Hubenov, 2008a).

Nilea rufiscutellaris (Zetterstedt, 1859)

Literature: BARANOv (1928). - Nearby countries: unverified.

Winthemia erythrura (Meigen, 1838)

Literature: LANGHoffer (1920). - Nearby countries: Bulgaria, Romania, Serbia \& Montenegro*, Slovenia (Tschorsnig et al., 2004; Hubenov, 2008a, 2008b).

Winthemia variegata (Meigen, 1824) [= Winthemia nigrithorax (Egger, 1861)]

Literature: BARANov (1928). - Nearby countries: Bulgaria, Romania, Serbia \& Montenegro*, Slovenia (Tschorsnig et al., 2004; Hubenov, 2008a, 2008b).

\section{Phasiinae}

Hemyda obscuripennis (Meigen, 1824) [= Phania obscuripennis Meigen, 1824]

Literature: BARANOv (1928). - Nearby countries: Bulgaria, Romania, Serbia \& Montenegro* (Tschorsnig et al., 2004; Hubenov, 2008a, 2008b).

\section{TACHININAE}

\section{Cleonice nitidiuscula (Zetterstedt 1859)}

Literature: BARANov (1928). - Nearby countries: Bulgaria (Tschorsnig et al., 2004; Hubenov, 2008a, 2008b).

\section{Bithia modesta (Meigen, 1824)}

Literature: LANGHoffer (1920). - Nearby countries: Bulgaria, Greece, Macedonia, Romania, Serbia \& Montenegro* (Tschorsnig et al., 2004; Hubenov, 2008a, 2008b, 2015).

Hyperaea femoralis (Meigen, 1824)

Literature: Frauenfeld (1856); Schiner (1862); Langhoffer (1896); Thalhammer (1899); Strobl (1902; 1904); Baranov (1928). - Nearby countries: unverified.

Linnaemya haemorrhoidalis (Fallén, 1810)

Literature: LANGHOFFer (1920); BARANov (1928). - Nearby countries: Bulgaria, Greece (TsCHORSNIG et al., 2004; HuBENOv, 2008a, 2008b, 2015).

Loewia setibarba Egger, 1856

Literature: SтrовL (1902; 1904). - Nearby countries: Bulgaria, Romania (Tschorsnig et al., 2004; Hubenov, 2008a, 2008b).

Lydina aenea (Meigen, 1824) [= Tachina aenea Meigen, 1824]

Literature: Frauenfeld (1856). - Nearby countries: Albania, Bulgaria, Greece, Romania, Serbia \& Montenegro*, Slovenia (Tschorsnig et al., 2004; Hubenov, 2008a, 2008b). 
Macquartia grisea (Fallén, 1810)

Literature: SCHINER (1862). - Nearby countries: Bosnia \& Herzegovina, Bulgaria, Romania, Serbia \& Montenegro* (Tschorsnig et al., 2004; Hubenov, 2008a, 2008b, 2015).

Siphona cristata (Fabricius, 1805)

Literature: LANGHOFFer (1920); BARANov (1928). - Nearby countries: Albania, Bulgaria, Greece, Macedonia (Tschorsnig et al., 2004; Hubenov, 2008a, 2015).

Siphona maculata Stæger, 1849

Literature: Thalhammer (1899); Baranov (1928). - Nearby countries: Bulgaria (Tschorsnig et al., 2004; Hubenov, 2008a, 2008b).

Solieria inanis (Fallén, 1810)

Literature: LANGHoffer (1920). - Nearby countries: Bosnia \& Herzegovina, Romania, Slovenia (Tschorsnig et al., 2004; Hubenov, 2008a, 2008b).

Doubtful taxa (Nomina dubia)

Cylindromyia nigrita (Meigen, 1824) [= Ocyptera nigrita Meigen, 1824]

Literature: Frauenfeld (1856).

Graphogaster maculiventris Stein, 1924

Literature: STEIN (1924).

Leucostoma brevis (Rossi, 1790)

Literature: Strobl (1893; 1902; 1904); ThalHammer (1899).

*Published sources provide a cumulative number of species, consisting of a former state that comprised Serbia, Montenegro and Kosovo. 